( С Д.Г. Бельцевич, Е.А. Трошина, Г.А. Мельниченко, Н.М. Платонова, Д.О. Ладыгина, А. Шевэ*

Национальный медицинский исследовательский центр эндокринологии, Москва, Россия

Более широкое применение и техническое совершенствование процедур визуализации брюшной полости в последние годы привело к все более частому обнаружению образований надпочечников - инциденталом, требующих, в свою очередь, исключения гормональной активности и злокачественности.

Клинические рекомендации - это основной рабочий инструмент практикующего врача, как специалиста, так и врача узкой практики. Лаконичность, структурированность сведений об определенной нозологии, методов ее диагностики и лечения, базирующихся на принципах доказательной медицины, позволяют в короткий срок дать тот или иной ответ на интересующий вопрос специалисту, добиваться максимальной эффективности и персонализации лечения. Эти клинические рекомендации включают данные о распространенности, этиологии, рентгенологических особенностях и оценке гормонального статуса инциденталом надпочечников. Кроме того, настоящие клинические рекомендации содержат информацию о показаниях к хирургическому лечению, послеоперационной реабилитации и последующем наблюдении.

КЛЮчЕВЫЕ СЛОВА: надпочечник; инциденталома; клинические рекомендации.

\title{
DRAFT OF THE CLINICAL PRACTICE GUIDELINES “ADRENAL INCIDENTALOMA"
}

(c) Dmitriy G. Beltsevich, Ekaterina A. Troshina, Galina A. Melnichenko, Nadezhda M. Platonova, Daria O. Ladygina, Anastassia Chevais*

Endocrinology Research Centre, Moscow, Russia

The wider application and technical improvement of abdominal imaging procedures in recent years has led to an increasingly frequent detection of adrenal gland masses - adrenal incidentaloma, which have become a common clinical problem and need to be investigated for evidence of hormonal hypersecretion and/or malignancy.

Clinical guidelines are the main working tool of a practicing physician. Laconic, structured information about a specific nosology, methods of its diagnosis and treatment, based on the principles of evidence-based medicine, make it possible to give answers to questions in a short time, to achieve maximum efficiency and personalization of treatment. These clinical guidelines include data on the prevalence, etiology, radiological features and assessment of hormonal status of adrenal incidentalomas. In addition, this clinical practice guideline provides information on indications for surgery, postoperative rehabilitation and follow-up.

KEYWORDS: adrenal gland; incidentaloma; guidelines.

\section{СПИСОК СОКРАЩЕНИЙ}

\section{$\mathrm{A} \Gamma$}

- артериальная гипертензия

АКТГ - адренокортикотропный гормон

АKР - адренокортикальный рак

AMPK - антагонисты минералокортикоидных рецепторов

АРC - альдостерон-рениновое соотношение

ВДКН - врожденная дисфункция коры надпочечников

ГНО - гормонально-неактивные опухоли

МСКТ - мультиспиральная компьютерная томография

MPT - магнитно-резонансная томография

$\mathrm{HH}$ - надпочечниковая недостаточность

ОТ - окружность талии

ПГА - первичный гиперальдостеронизм

ПТД1 - ночной подавляющий тест с 1 мг дексаметазона

ПЭТ - позитронно-эмиссионная томография

СK - синдром Кушинга

УзИ - ультразвуковое исследование

ФАПК - функционально автономная продукция кортизола

ФДГ - фтордезоксиглюкоза

ФХЦ - феохромоцитома

ФХЦ/ПГ — феохромоцитома/параганглиома

HU - единица Хаунсфилда

MIBG - метайодбензилгуанидин

\section{ТЕРМИНЫ И ОПРЕДЕЛЕНИЯ}

Адренокортикальный рак (АКР) - редкая злокачественная опухоль коры надпочечников, характеризующаяся, как правило, поздним сроком выявления, агрессивностью клинического течения и неблагоприятным лечебным прогнозом.

Первичный гиперальдостеронизм (ПГА) - это совокупность нарушений, при которых производство альдостерона значительно повышено, относительно автономно от основных регуляторов ренин-ангиотензиновой системы и не подавляется нагрузкой натрием.

Острая надпочечниковая недостаточность - симптомокомплекс, обусловленный резким снижением или полным прекращением функциональной деятельности коры надпочечников.

Функционально автономная продукция кортизола (ФАПК) - патологическое состояние, характеризующееся АКТГ-независимой избыточной продукцией кортизола и отсутствием классических «больших признаков» гиперкортицизма.

Феохромоцитома/параганглиома (ФХЦ/ПГ) - опухоль симпатоадреналовой системы из хромаффинных клеток надпочечниковой или вненадпочечниковой локализации, как правило, избыточно секретирующая катехоламины. 


\section{1. КРАТКАЯ ИНФОРМАЦИЯ.}

\section{1. ОПРЕДЕЛЕНИЕ.}

Инциденталома (случайно выявленное образование) надпочечника - собирательный феномен, требующий нозологической конкретизации. Термин «инциденталома» включает разнообразные по морфологии опухоли более 1 см в диаметре, случайно выявленные при радиологическом обследовании.

Определяемая опухоль надпочечника не ограничена никакими функциональными рамками и строгими критериями тканевой принадлежности. Выявленное образование может оказаться как гормонально-неактивным, так и активно производящим различные гормоны, может исходить из различных зон надпочечника или иметь неспецифичную органную принадлежность, может быть как злокачественным, так и доброкачественным [1].

К инциденталомам надпочечника не принято относить образования менее 1 см. Хотя это пороговое значение несколько произвольно, считается, что дальнейшего диагностического обследования требуют лишь образования более 1 см, если только не присутствуют клинические признаки, указывающие на избыточную продукцию гормонов [2].

\section{2. ЭТИОЛОГИЯ И ПАТОГЕНЕЗ.}

Этиология инциденталом надпочечников различна и включает доброкачественные и злокачественные образования, как происходящие из различных слоев коры надпочечников, так и имеющие вненадпочечниковую этиологию.

Гормонально-активные опухоли могут происходить из любого слоя надпочечников, среди них выделяют кортикостеромы, альдостеромы, феохромоцитомы/параганглиомы (ФХЦ/ПГ), андростеромы, а также опухоли со смешанной гормональной секрецией.

В свою очередь, гормонально-неактивные опухоли (ГНО) представлены аденомами, кистами, адренокортикальным раком (АКР), метастазами опухолей другой локализации. Значительно реже встречаются миелолипомы, ганглионейромы, нейробластомы, шванномы, липомы, гемангиомы и др.

В молодом возрасте наиболее частой причиной формирования непаразитарных кист являются травма и, как след- ствие, кровоизлияние в надпочечник. У взрослых/пожилых людей кровоизлияния в надпочечник могут быть ассоциированы с тромбоэмболической болезнью, коагулопатиями. Бактериальные, грибковые инфекции (гистоплазмоз), а также паразитарные поражения (эхинококкоз) могут сопровождаться формированием кист, которые иногда могут носить двусторонний характер поражения [3].

\section{3. эПИДЕМИология.}

«Эпидемия» инциденталом надпочечника напрямую связана с широким распространением и использованием визуализирующих инструментальных методов исследования: ультразвукового (УЗИ), компьютерной томографии (КТ) и магнитно-резонансной томографии (МРТ).

Сведения о заболеваемости и распространенности инциденталом надпочечников могут быть получены только на основе результатов визуализирующих методов исследований или данных аутопсии, поскольку по определению нет специфических клинических проявлений этих опухолей.

По данным сводной аутопсийной статистики, распространенность случайно выявленных опухолей надпочечника составляет в среднем 6\% [4-6]. По данным мультиспиральной КТ (МСКТ), «случайные» образования надпочечника выявляются приблизительно у 4\% обследованных пациентов [7]. В возрасте до 30 лет инциденталома встречается приблизительно у 0,2\% обследованных, однако в группе пациентов старше 70 лет частота выявления возрастает до 7\% (табл. 1) [8].

Представления о частоте выявления АКР при инциденталомах как потенциально наиболее опасного заболевания пересмотрены в связи с расширением клинической базы исследований и ужесточением требований к характеру и дизайну эпидемиологических исследований. Так, по данным W.F. Young и соавт. (2007), частота АКР оценивалась чуть более 4\% [8]. Позднее, согласно сводным результатам межклинического анализа большого числа пациентов в догоспитальных выборках, распространенность АКР среди инциденталом не превысила 1,9\% [9]. Довольно часто в публикациях фигурируют госпитальные выборки пациентов с инциденталомами, в которых доля больных с АКР, по понятным причинам, более высокая (10-15\%) [10,11].

Таблица 1. Встречаемость различного типа опухолей при случайном их выявлении (сводные данные литературы)

\begin{tabular}{|c|c|}
\hline Тип опухоли & Встречаемость \\
\hline Гормонально-неактивные аденомы & $60-65 \%$ \\
\hline Гормонально-активные аденомы (функционально-автономная продукция кортизола) & $8-10 \%$ \\
\hline Альдостерома & $1 \%$ \\
\hline Адренокортикальный рак & $1,9-4,5 \%$ \\
\hline ФХЦ/ПГ & $5-6 \%$ \\
\hline Метастатическое поражение & $2-4 \%$ \\
\hline Двусторонние опухоли & $15-17 \%$ \\
\hline Непаразитарные кисты & $5-7 \%$ \\
\hline Миелолипома & $2-5 \%$ \\
\hline Ганглионейрома, нейробластома, шваннома & $0,5-1,5 \%$ \\
\hline $\begin{array}{l}\text { Липома, лейомиома/саркома, паразитарная киста (эхинококк), гемангиома, гамартома, гематома, } \\
\text { тератома, гранулема различной этиологии, псевдонадпочечниковые образования (исходящие } \\
\text { из других органов) }\end{array}$ & раритет \\
\hline
\end{tabular}




\section{4. ОСОБЕННОСТИ КОДИРОВАНИЯ ЗАБОЛЕВАНИЯ ИЛИ СОСТОЯНИЯ (ГРУППЫ ЗАБОЛЕВАНИЙ ИЛИ СОСТОЯНИЙ) ПО МЕЖДУНАРОДНОЙ СТАТИСТИЧЕСКОЙ КЛАССИФИКАЦИИ БОЛЕЗНЕЙ И ПРОБЛЕМ, СВЯЗАННЫХ СО ЗДОРОВЬЕМ.}

В международной классификации болезней 10-го пересмотра (МКБ-10) инциденталомы надпочечников не выделены в отдельную нозологическую группу, так как являются собирательным клиническим термином.

D35 Доброкачественное новообразование других и неуточненных эндокринных желез:

- D35.0 - надпочечника.

\section{5. КЛАССИФИКАЦИЯ.}

В литературе предлагаются различные варианты систематизации инциденталом надпочечников, однако ввиду гетерогенности этиологии новообразований, использования различных методов диагностики при первичном выявлении в настоящий момент общепринятая классификация инциденталом надпочечников отсутствует.

Наиболее полной считается классификация инциденталом, предложенная американскими исследователями M. Gross и B. Shapiro (1993):

- образования, исходящие из коры надпочечника: аденома, узловая гиперплазия, карцинома;

- образования, исходящие из мозгового слоя: феохромоцитома, ганглионеврома, ганглионейробластома;

- другие поражения надпочечников: миелолипома, нейрофиброма, гамартома, тератома, ксантоматоз, амилоидоз, киста, гематома, гранулема, лейомиома, лейомиосаркома;

- метастазы: рака молочной железы, легких, почек, лимфомы и др.;

- псевдонадпочечниковые поражения: образования, исходящие из почек, поджелудочной железы, селезенки, лимфатических узлов, сосудов;

- технические артефакты [12].

Основываясь на данных радиологических исследований, образования надпочечниковой локализации можно классифицировать в зависимости от их КТ-семиотики:

- доброкачественный КТ-фенотип: опухоль до 4 см, однородной структуры, низкой нативной плотности (менее 10-15 HU):

- неопределенный КТ-фенотип: опухоли высокой плотности менее 4 см; опухоли низкой плотности более 4 см; опухоли мозаичной плотности;

- злокачественный КТ-фенотип: опухоль более 4 см высокой нативной КТ-плотности; любая опухоль с признаками инвазивного роста в соседние органы, опухолевого или сосудистого тромбоза [13].

\section{6. КЛИНИЧЕСКАЯ КАРТИНА.}

Исходя из определения инциденталомы, данное образование выявлено случайно и не сопровождается яркими клиническими проявлениями нарушения функции надпочечников. Симптомы, как правило, отсутствуют.

По данным ряда исследований, несмотря на отсутствие типичных клинических проявлений, у пациентов с функционально-автономной продукцией кортизола значительно чаще выявляются такие коморбидные состояния, как избыточная масса тела, артериальная гипертензия (АГ), сахарный диабет (СД), дислипидемия, репродуктивные расстройства и снижение минеральной плотности кости (вплоть до остеопороза) [14-17].

Часть пациентов при детальном расспросе могут предъявлять неспецифические жалобы (боль в проекции надпочечника, быстрая утомляемость, слабость, изменение массы тела и др.), которые не позволяют по клинической картине судить о функциональной активности опухоли или ее злокачественном потенциале. У части пациентов может наблюдаться субфебрилитет, который наиболее часто ассоциирован с развитием интоксикационного синдрома при злокачественных опухолях или с инфекционным поражением. Тем не менее симптомы, традиционно ассоциируемые с онкологическим заболеванием (похудение, повышенное потоотделение в ночное время, выраженная слабость, лихорадка), как правило, нехарактерны для АКР.

\section{2. ДИАГНОСТИКА, МЕДИЦИНСКИЕ ПОКАЗАНИЯ И ПРОТИВОПОКАЗАНИЯ К ПРИМЕНЕНИЮ МЕТОДОВ ДИАГНОСТИКИ.}

Критерии установления диагноза: случайно выявленная при радиологическом обследовании опухоль надпочечника.

- При выявлении опухоли надпочечника размером более $1 \mathrm{~cm}$ рекомендовано оценить злокачественный потенциал опухоли и возможность гормональной гиперсекреции.

Уровень убедительности рекомендаций А (уровень достоверности доказательств 2).

Комментарии. Крайне важно исключить злокачественный характер новообразования надпочечника, поскольку тактика лечения в случае его подтверждения будет принципиально отличаться вне зависимости от гормональной активности образования. Злокачественные образования могут потребовать срочного хирургического вмешательства или других методов лечения, а промедление может причинить вред.

Несмотря на то что при первичном обращении пациенты не будут предъявлять специфических жалоб, ассоциированных с гиперпродукцией гормонов, все пациенты должны пройти тщательное физикальное обследование с подробным сбором анамнеза, поскольку затем будет определен объем лабораторной диагностики, которая позволит выявить/исключить гормональные, метаболические и электролитные нарушения, выявить/исключить первичный гиперальдостеронизм, функционально-автономную продукцию кортизола, «немую» феохромоцитому.

\section{1. ЖАЛОБЫ И АНАМНЕЗ.}

Наличие и характер жалоб в большей степени зависят от наличия или отсутствия гормональной активности опухоли. При расспросе пациента необходимо уточнять наличие АГ той или иной степени выраженности (медикаментозно-корригируемая; пароксизмального типа; стойкая, резистентная к терапии), нарушений углеводного обмена (нарушенная толерантность к глюкозе, СД) - жажда, полиурия, колебания веса; симптомов, косвенно указывающих на нарушение минерально-костного обмена 
(низкотравматические переломы, снижение роста и др.), прибавку массы тела, мышечную и общую слабость.

При сборе анамнеза следует обратить внимание на следующие признаки:

- наличие онкологического анамнеза;

- наличие травм поясничной области в анамнезе;

- наличие отягощенной наследственности;

- наличие врожденной дисфункции коры надпочечников.

В некоторых случаях симптомами могут быть боли В поясничной области, субфебрильная температура.

\section{2. ФИЗИКАЛЬНОЕ ОБСЛЕДОВАНИЕ.}

Общий осмотр подразумевает оценку общего физического состояния, роста и массы тела, распределения подкожно-жировой клетчатки, окружности талии (ОТ). Пальпаторно можно определить лишь образования достаточно крупного размера.

\section{3. ЛАБОРАТОРНЫЕ ДИАГНОСТИЧЕСКИЕ ИССЛЕДОВАНИЯ.}

Проведение лабораторных тестов необходимо не только для установления факта наличия гормональных нарушений, но и для определения степени выраженности гормональной активности опухолевого образования, которая будет в ряде случаев иметь принципиальное значение в выборе тактики ведения.

- Исследование гормональной активности опухоли надпочечника строго регламентировано, и его результаты рекомендовано учитывать при выборе тактики ведения, планировании предоперационной подготовки, объема операции, последующего наблюдения за больным.

Уровень убедительности рекомендаций А (уровень достоверности доказательств 2).

Комментарии. В связи с увеличивающейся распространенностью и общедоступностью топических методов диагностики (МСКТ, МРТ, УЗИ) за последние четыре десятилетия отмечается увеличение числа инциденталом надпочечников.

Несмотря на отсутствие клинической симптоматики и манифестных признаков гормональной активности, требуется лабораторное подтверждение ее наличия или отсутствия. В 85\% случаев инциденталомы являются ГНО [18].

При случайно выявленных опухолях частота встречаемости ФХЦ оценивается в 5-6\%, при этом на «бессимптомные» формы приходится более 50\% случаев [1]. Иногда встречается обратная ситуация: яркая клиническая картина гиперпродукции определенного гормона нацеливает врача на «очевидный» диагноз, казалось бы, не требующий тщательной гормональной диагностики. Зачастую морфологически опухоль имеет иную природу, отличную от «очевидного» диагноза. Так, в литературе неоднократно описаны ФХЦ, проявляющиеся не только гиперкатехоламинемией, но и эктопированной продукцией кортикотропина (АКТГ), сопровождающейся «большими» признаками гиперкортицизма [19].

Описаны опухоли, протекающие с классическими пароксизмальными кризами, характерными для ФХЦ, которые в действительности оказывались «псевдофеохро- моцитомами» - гормонально-неактивными аденомами коры надпочечника или АКР $[18,20]$.

Среди инциденталом нередко выявляется феномен функционально автономной продукции кортизола (ФАПК) - 8-12\% наблюдений. Однако, несмотря на бессимптомное течение заболевания, длительное влияние избытка кортизола в части случаев может оказывать медленный, но, тем не менее, разрушающий эффект на многие системы организма человека [21, 22]. В метаанализе Elhassan и соавт. отмечается повышенный кардиоваскулярный риск у пациентов с ФАПК. Также прибавка в массе тела и СД2 наблюдались в два раза чаще у пациентов с ФАПК, чем у пациентов с ГНО [23].

С целью структуризации излагаемой информации, с учетом гормональной активности рекомендации будут разделены на подразделы: «эндогенный гиперкортицизм», «феохромоцитома», «первичный гиперальдостеронизм», «гиперандрогения».

\section{Эндогенный гиперкортицизм.}

При опухолях надпочечников, сопровождающихся выраженной гиперпродукцией кортизола, достаточно редкой является ситуация истинной инциденталомы, когда образование выявлено случайно. При развернутой клинической картине гиперкортицизма, как правило, топические исследования проводятся целенаправленно. Проблема инциденталом надпочечника и гиперкортицизма наиболее актуальна с точки зрения ФАПК [24].

Недооценка наличия АКТГ-независимого гиперкортицизма (манифестного или субклинического) при инциденталоме надпочечника связана с высоким риском развития послеоперационной острой надпочечниковой недостаточности (НН), которая в случае несвоевременного лечения ассоциирована с высоким риском летального исхода. Это является основной причиной, из-за которой диагностика ФАПК является строго обязательной. Трудности своевременной диагностики НН вызваны неспецифичностью клинических проявлений (гипотония, слабость, отсутствие аппетита, тошнота/рвота, вздутие живота, подъемы температуры), более того, часто симптомы «маскируются» под другие осложнения (кровотечение, интоксикация, острый инфаркт миокарда, кишечная непроходимость, послеоперационные инфекции и т.д.). В основе патогенеза данного явления лежит подавление функции контралатерального надпочечника, приводящее к атрофии его коры.

- Всем пациентам с выявленной опухолью надпочечника рекомендовано определение кортизола в ранние утренние часы на фоне подавляющего теста с 1 мг дексаметазона.

Интерпретация результатов:

- кортизол менее 50 нмоль/л (1,8 мкг/дл) - эндогенный гиперкортицизм исключен;

- кортизол 51-139 нмоль/л (1,8-5 мкг/дл) - подозрение на эндогенный гиперкортицизм;

- кортизол более 140 нмоль/л (5 мкг/дл) - эндогенный гиперкортицизм высоковероятен.

Уровень убедительности рекомендаций В (уровень достоверности доказательств 2).

Комментарии. Существующие международные клинические рекомендации в отношении диагностики гиперкортицизма имеют достаточную доказательную базу $[21,25]$. В качестве первичной диагностики эндогенного 
Таблица 2. Лекарственные препараты, влияющие на уровни кортизола в крови/суточной моче и изменяющие результаты фармакологических тестов, проводимых для диагностики эндогенного гиперкортицизма [25]

\section{Действие}

Лекарственный препарат

- Фенобарбитал

- Фенитоин

Лекарственные препараты, ускоряющие метаболизм дексаметазона на счет индукции фермента СYР3А4 печени

- Карбамазепин

- Рифапентин

- Рифампицин

- Этосуксимид

- Пиоглитазон

- Итраконазол

- Ритонавир

- Флуоксетин

- Дилтиазем

- Циметидин

Лекарственные препараты, повышающие уровень кортизол-связывающего белка (ложноположительное повышение кортизола в крови)

- Эстрогены

- Митотан

Лекарственные препараты, ингибирующие 11HSD2 (ложноположительное повышение кортизола в суточной моче)
- Препараты лакрицы

- Карбеноксолон
Лекарственные препараты, имеющие другие различные механизмы действия 11-гидроксистероиддегидрогеназы 2-го типа (ложноположительное повышение кортизола в суточной моче)
- Карбамазепин

- Фенофибрат

- Синтетические ГКС гиперкортицизма могут быть использованы различные методы: ночной подавляющий тест с 1 мг дексаметазона (ПДТ1), исследование уровня суточной экскреции кортизола мочой (двукратное определение), исследование уровня кортизола в слюне в 23:00-00:00 (двукратное определение), малая проба Лиддла (двухдневная проба с 2 мг дексаметазона). Однако, к сожалению, ни один тест не обладает высокой специфичностью, в связи с чем возможны ложноположительные результаты [25].

На основании патофизиологических особенностей, простоты, доступности, высокой чувствительности и факта включения в диагностические алгоритмы большинства исследований ПдТ1 рекомендован в качестве первичного метода диагностики эндогенного гиперкортицизма.

В основе ПДТ1 лежит подавление секреции АКТГ и, как следствие, снижение синтеза кортизола в ответ на прием супрафизиологической дозы глюкокортикоидов. Как следует из определения ФАПК, продукция кортизола не зависит от стимула АКТГ, а носит автономный характер, следовательно, при назначении низких доз дексаметазона подавления кортизола не происходит. ПдТ1 отличается простотой при проведении в амбулаторных условиях: 1 мг дексаметазона принимается между 23 и 24 ч, на следующее утро в интервале между 8 и 9 ч определяется уровень кортизола в крови. Более высокие дозы (1,5 или 2 мг) не улучшают точность теста.

Чувствительность в выявлении ФАПК для снижения кортизола на фоне проведения ПтД1 при cut-off 130-140 нмоль/л составляет 80\%, при cut-off 50 нмоль/л приближается к 100\%, в связи с чем это пороговое значение имеет высокую отрицательную прогностическую ценность. Закономерно, что при повышении чувствительности значительно снижается специфичность те- ста. Специфичность ПДТ1 для значений 50-140 нмоль/л не превышает $80 \%$, что означает, что при этих значениях у $20 \%$ здоровой популяции будет отсутствовать подавление кортизола. Даже при превышении концентрации 140 нмоль/л специфичность составляет всего 95\% [9, 26].

Также необходимо учитывать лекарственные препараты и/или состояния, которые могут повлиять на результат данного теста (табл. 2), по возможности, рекомендуется их отмена за 6 нед до исследования.

Могут потребоваться дополнительные биохимические тесты для подтверждения секреторной автономной продукции кортизола и оценки степени ее выраженности. Для определения возможности динамического наблюдения пациента большое значение имеют возраст, наличие сопутствующих заболеваний, потенциально связанных с избыточной продукцией кортизола (метаболический синдром, ожирение, СД, АГ, остеопороз).

Возможны другие способы диагностики эндогенного гиперкортицизма (кортизол суточной мочи, кортизол в вечерней слюне). Однако при инциденталомах надпочечников данные исследования обладают меньшей чувствительностью в сравнении с ПДТ1 и не рекомендуются в качестве скринингового метода.

- При отсутствии после проведения подавляющего дексаметазонового теста физиологической супрессии уровня кортизола в качестве теста, подтверждающего АКТГ-независимую природу гиперкортицизма, предлагается использовать определение базального уровня (8:00-9:00) АКТГ.

Уровень убедительности рекомендаций В (уровень достоверности доказательств 3).

Комментарий. С целью уменьшения числа ложноположительных результатов, в качестве верифицирующего теста, рекомендовано определение утреннего уровня АКТГ. 
Подавление уровня АКТГ менее 1 пг/мл в сочетании с отсутствием подавления утреннего кортизола при проведении ПдТ1 с высокой долей вероятности подтверждает диагноз АКТГ-независимого гиперкортицизма у пациентов с опухолью надпочечников. Измерение АКТГ не является методом первичной диагностики, однако может служить подтверждающим признаком ФАПК. При отсутствии подавления уровня АКТГ ниже нижней границы референса (5 пг/мл) диагноз ФАПК маловероятен $[27,28]$. Чувствительность и специфичность 79 и $85 \%$ соответственно [18]. Дискутабелен уровень базального АКТГ в качестве верифицирующего ФАПК теста от менее 1 до 5 пг/мл - количество публикаций недостаточно для формирования доказательной рекомендации. Следует обращать внимание на необходимость оценки уровня АКТГ до или через 72 ч и более после проведения ПдТ1 во избежание получения ложноположительных результатов (из-за возможной остаточной концентрации дексаметазона в крови).

Автономная секреция кортизола ведет к угнетению синтеза АКТГ гипофизом и, как следствие, отсутствует стимуляция здоровой ткани коры надпочечников, что ведет к ее атрофии. В систематическом обзоре Dalmazi и соавт. от 2014 г. исследовалась функция надпочечников после односторонней адреналэктомии при синдроме Кушинга (СК). Критериями включения, помимо отсутствия подавления кортизола в ходе ПДТ1 (<138 нмоль/л), был супрессированный или нормальный уровень АКТГ (>5 пг/мл) и/или дополнительный верифицирующий анализ - кортизол суточной мочи, ночной сывороточный кортизол, повышенный уровень дегидроэпиандростерона ДГЭА для группы с «субклиническим гиперкортицизмом» и наличие «больших» клинических признаков СК, неопределяемого и подавленного уровня АКТГ для группы с манифестным СК. Выявлена положительная корреляция сниженного уровня АКТГ с развитием $\mathrm{HH}$ в послеоперационном периоде, однако уровень cut-off снижения на данный момент не определен [29]. В последующих работах также отмечается взаимосвязь между сниженным уровнем АКТГ и длительностью НН, соответственно, чем ниже уровень АКТГ, тем продолжительней $\mathrm{HH}[30,31]$.

Определение уровня АКТГ в условиях подавления уровня кортизола или изолированное определение данного показателя нецелесообразно и может привести к диагностическим ошибкам [28].

Анализ уровня АКТГ в утренние часы служит также для дифференциальной диагностики между СК центрального и надпочечникового генеза. При болезни Иценко-Кушинга показатель АКТГ будет превышать отметку в 10 пг/мл [25].

- Не рекомендовано рассматривать ФАПК как состояние с высоким риском развития манифестного СК.

Уровень убедительности рекомендаций А (уровень достоверности доказательств 2).

Комментарий. В публикациях до 2012 г. инциденталома надпочечника с проявлениями субклинического гиперкортицизма (принятый в то время термин) считалась фактором риска развития манифестного гиперкортицизма в течение 5 лет с вероятностью 25\%. Однако данные более поздних исследований не подтвердили эту гипотезу $[8,9,14]$.
По данным метаанализа британской группы, риск развития манифестного гиперкортицизма у пациентов как с ФАПК, так и с ГНО крайне мал и составляет менее 0,1\%. Средний период наблюдения составил 49,6 мес, среди 2745 человек, вошедших в исследование, лишь у 6 зафиксировано развитие манифестного СК. В 19 исследованиях, включающих 2083 пациента, изучался риск развития гормональной активности образования, в частности ФАПК, у пациентов с первоначально ГНО, что было выявлено в 4,3\%. Средний период наблюдения составил 50,3 мес [23]. В то же время, по данным проспективного исследования финских исследователей, наблюдавших в течение 5 лет 56 пациентов с ГНО, ни у одного пациента не было зафиксировано изменения гормонального статуса опухоли [32].

\section{Феохромоцитома/параганглиома}

ФХЦ составляют около 5-7\% инциденталом надпочечников. На современном этапе развития диагностических методов многие авторы отмечают, что не менее 50\% ФХЦ изначально были выявлены как инциденталомы, и только около половины этих наблюдений сопровождались АГ [1].

Недооценка наличия катехоламин-секретирующей опухоли связана с высоким риском периоперационного развития высокоамплитудных гипертензивных кризов, отека легких, фатальных аритмий, синдрома «неуправляемой гемодинамики» и внезапной сердечной смерти. Даже если клиническое течение ФХЦ на дооперационном этапе было «бессимптомным», высокий уровень циркулирующих катехоламинов может привести ко всем вышеперечисленным осложнениям [33].

- При инциденталоме надпочечника в качестве метода первичной диагностики ФХЦ/ПГ рекомендовано определение свободных метанефринов плазмы или фракционированных метанефринов суточной мочи.

Уровень убедительности рекомендаций А (уровень достоверности доказательств 2).

Комментарий. В качестве лабораторного метода исследования метилированных производных катехоламинов (метанефрина, норметанефрина и метокситирамина) в биологических жидкостях предлагается использовать жидкостную хроматографию электрохимическими, флуорометрическими детекторами (LC-ECD) или тандемными масс-спектрометрами (LC-MS/MS). Возможно использование иммунологического метода, однако диагностическая чувствительность этого метода является более низкой по сравнению с вышеописанными [34, 35].

При исследовании уровня метанефринов плазмы забор крови необходимо проводить после 30-минутного положения лежа на спине. Это обусловлено быстрым клиренсом метаболитов катехоламинов и выраженным влиянием активации симпатической нервной системы при вертикальном положении на стимуляцию выработки норадреналина [36].

При сравнительном анализе различных методов диагностики ФХ/ПГ выявлено, что наибольшей диагностической чувствительностью обладает анализ метанефринов в крови при заборе в положении лежа, чем в положении сидя, 95\% и 89\% соответственно, в то время как этот же показатель составил 93\% при анализе суточной мочи на метилированные катехоламины. Также исследование 
Таблица 3. Лекарственные препараты, влияющие на показатели метанефринов плазмы и мочи (ложноположительный результат)

\begin{tabular}{|c|c|c|c|c|}
\hline & \multicolumn{2}{|c|}{ Плазма } & \multicolumn{2}{|c|}{ Моча } \\
\hline & Норметанефрин & Метанефрин & Норметанефрин & Метанефрин \\
\hline Ацетаминофен & $\uparrow \uparrow$ & - & $\uparrow \uparrow$ & - \\
\hline Лабеталол & - & - & $\uparrow \uparrow$ & $\uparrow \uparrow$ \\
\hline Соталол & - & - & $\uparrow \uparrow$ & $\uparrow \uparrow$ \\
\hline а-Метилдопа & $\uparrow \uparrow$ & - & $\uparrow \uparrow$ & - \\
\hline $\begin{array}{l}\text { Трициклические } \\
\text { антидепрессанты }\end{array}$ & $\uparrow \uparrow$ & - & $\uparrow \uparrow$ & - \\
\hline Буспирон & - & $\uparrow \uparrow$ & - & $\uparrow \uparrow$ \\
\hline Феноксибензамин & $\uparrow \uparrow$ & - & $\uparrow \uparrow$ & - \\
\hline Ингибиторы МАО & $\uparrow \uparrow$ & $\uparrow \uparrow$ & $\uparrow \uparrow$ & $\uparrow \uparrow$ \\
\hline Симпатомиметики & $\uparrow$ & $\uparrow$ & $\uparrow$ & $\uparrow$ \\
\hline Кокаин & $\uparrow \uparrow$ & $\uparrow$ & $\uparrow \uparrow$ & $\uparrow$ \\
\hline Сульфасалазин & $\uparrow \uparrow$ & - & $\uparrow \uparrow$ & - \\
\hline Леводопа & $\uparrow$ & $\uparrow$ & $\uparrow \uparrow$ & $\uparrow$ \\
\hline
\end{tabular}

$\uparrow \uparrow —$ высокое увеличение; $\uparrow$-умеренное увеличение; «-» - без увеличения

метанефринов в крови имеет большую специфичность, чем анализ суточной мочи, - 95\% против 90\% [34]. Однако забор крови в положении лежа отнимает больше времени и приводит к дополнительным затратам. В случаях, когда по какой-то причине выполнение этой рекомендации невозможно, альтернативным вариантом является исследование фракционированных метанефринов мочи [36, 37].

Определение адреналина и норадреналина в биологических жидкостях не рекомендовано в связи с крайне низкой чувствительностью и специфичностью данного метода.

- Всем пациентам с положительным результатом тестирования необходимо углубленное обследование для исключения или подтверждения ФХЦ/ПГ.

Уровень убедительности рекомендаций В (уровень достоверности доказательств 2).

Комментарии. Трехкратное повышение уровня норметанефринов и двукратное повышение метанефринов подтверждает наличие у пациента ФХ/ПГ и не требует других подтверждающих тестов [33, 38].

Наибольшую диагностическую трудность представляет интерпретация результатов, находящихся В «серой зоне», - не достигающих нужного трехкратного повышения. Это составляет около $25 \%$ случаев впоследствии подтвержденной другими методами ФХЦ/ПГ. Чаще всего причиной искажения результатов является нарушение техники проведения исследования, что требует повторного определения. Выраженный физиологический стресс, связанный, например, с тяжелым заболеванием, также может быть причиной значительного повышения уровня метанефринов в крови и суточной моче [39]. Также необходимо помнить о ряде препаратов, которые могут искажать результаты исследований (например, ацетаминофен, мезаламин, сульфасалазин в LC-ECD методе или трициклические антидепрессанты, влияющие непосредственно на катехоламины) (табл. 3) $[40,41]$.

При сомнительных результатах гормональных исследований для верификации диагноза ФХЦ целесообразно проведение сцинтиграфии с ${ }^{123} \mathrm{I}-\mathrm{MIBG}$, чувствительность которой достигает 93-100\% при специфичности $89 \%[42,43]$. Возможна и выжидательная тактика повторное исследование уровня метанефринов через 6 мес [33].

- При доброкачественном КТ-фенотипе инциденталомы надпочечника (однородная структура образования низкой нативной плотности менее $10 \mathrm{HU}$ ) у нормотензивных пациентов диагностика ФХЦ не рекомендована.

Уровень убедительности рекомендаций А (уровень достоверности доказательств 2).

Комментарии. Среднее значение нативной плотности ФХЦ варьирует от 30 до 35 HU. При низких показателях нативной плотности (<10 HU) при МСКТ вероятность ФХЦ крайне мала и составляет 0,5\%. Buitenwerf и соавт. ретроспективно оценили КТ-картину 222 гистологически подтвержденных ФХЦ, лишь у 1 выявлена низкая нативная плотность. В аналогичной работе Canu и соавт. обнаружено 2 случая ФХЦ с низкой нативной плотностью из 376 гистологически подтвержденных катехоламинпродуцирующих опухолей. Чувствительность данного метода приближена к абсолютным значениям $(99,6 \%)$ [32, 44, 45]. На основании данных исследований ставится под сомнение необходимость исследования метанефринов у нормотензивных пациентов с низкой плотностью инциденталомы надпочечников. В качестве верифицирующего теста при получении «серой зоны» метанефринов предлагается оценка нативной плотности тканевого компонента опухоли надпочечника при МСКТ.

Исключением являются кистозные образования, которые могут обладать различной плотностью (5-25HU), однако их патогномоничным признаком будет являться отсутствие накопления контрастного вещества при МСКТ. Кистозные формы ФХЦ достаточно редки и не всегда сопровождаются клинической симптоматикой. Это определяет потенциальную опасность пропущенного диагноза. 
Алгоритм диагностики и лечения ФХЦ/ПГ подробным образом изложен в клинических рекомендациях по данной нозологии.

Первичный гиперальдостеронизм.

Вторичные АГ, выявляемые у 25\% пациентов с эндокринной патологией, как правило, характеризуются более быстрым дебютом, агрессивным течением и резистентностью к проводимой антигипертензивной терапии. Выявление ПГА важно не только из-за высокой распространенности, но и потому, что пациенты с ПГА имеют более высокую сердечно-сосудистую заболеваемость и смертность, чем пациенты с эссенциальной АГ [46]. При рандомизированном двукогортном исследовании Milliez и соавт. получили данные по встречаемости нежелательных сердечно-сосудистых событий среди данных групп пациентов, так, в группе с ПГА распространенность ОИМ составила 12,9\% против $3,4 \%$ у пациентов с эссенциальной АГ, нарушения ритма фиксировались в 7,3\% против 0,6\% соответственно [47].

Целесообразно однократно провести тестирование для выявления случаев ПГА у всех пациентов с артериальной гипертензией. Обследование для постановки диагноза и определения формы ПГА представляет собой последовательность из трех этапов, включающих скрининговые или первичные тесты, подтверждающие тесты и определение латерализации автономной секреции альдостерона (дифференциальная диагностика односторонних и двусторонних форм ПГА) [48].
- При сочетании инциденталомы надпочечника и АГ рекомендована первичная диагностика ПГА: определение уровня альдостерона, ренина плазмы и калия сыворотки. Положительным первичным тестом на ПГА можно считать сочетание уровня альдостерона в верхней половине референса (>10 нг/дл/100 пг/мл/277 пмоль/л) и активности ренина $<1$ нг/мл/ч (или прямого ренина ниже референсного значения).

Уровень убедительности рекомендаций С (уровень достоверности доказательств 4).

Комментарии. Первичная диагностика ПГА включает определение уровня альдостерона, ренина и калия в сыворотке крови. Забор крови осуществляют до 10 ч утра на фоне либерализованной по соли диеты, при этом пациенту не обязательно приходить натощак. В течение 2 ч до анализа желательно нахождение пациента в вертикальном положении, а непосредственное взятие крови проводится в положении сидя. Специальная подготовка к первичной лабораторной диагностике ПГА не требуется.

Пациент может принимать любые антигипертензивные препараты, в том числе антагонисты минералокортикоидных рецепторов (АМКР). Список лекарственных препаратов, способных влиять на ренин-ангиотензин-альдостероновую ось у пациентов без ПГА, обширен (табл. 4). Однако клиницистам важно понимать, что, хотя лекарственные препараты, используемые для лечения АГ, потенциально могут вызывать

Таблица 4. Лекарственные препараты и состояния, которые могут оказывать влияние на уровни альдостерона и активности ренина плазмы/ прямого ренина

\begin{tabular}{|c|c|c|}
\hline Условия & Альдостерон & АРП и/или ПКР \\
\hline \multicolumn{3}{|c|}{ Медикаменты } \\
\hline Бета-блокаторы & $\downarrow$ & $\downarrow \downarrow$ \\
\hline Центральные альфа-адреномиметики & $\downarrow$ & $\downarrow \downarrow$ \\
\hline $\mathrm{H} \Pi \mathrm{BC}$ & $\downarrow$ & $\downarrow \downarrow$ \\
\hline Калийтеряющие диуретики & $\uparrow-$ & $\uparrow \uparrow$ \\
\hline Калийсберегающие диуретики & $\uparrow$ & $\uparrow \uparrow$ \\
\hline Ингибиторы АПФ & $\downarrow$ & $\uparrow \uparrow$ \\
\hline Блокаторы АТ1-рецепторов & $\downarrow$ & $\uparrow \uparrow$ \\
\hline Блокаторы кальциевых каналов (дигидропиридины) & $\downarrow-$ & $\uparrow$ \\
\hline Ингибиторы ренина & $\downarrow$ & $\downarrow(\mathrm{AP} \Pi) \uparrow(\Pi K \mathrm{P})$ \\
\hline Эстрогенсодержащие препараты & - & $-($ АРП) $\downarrow(П К Р)$ \\
\hline
\end{tabular}

\begin{tabular}{lccc}
\hline \multicolumn{1}{l}{ Уровень калия } & & \\
\hline Гипокалиемия & $\downarrow$ & $\uparrow-$ \\
Гиперкалиемия & & $\uparrow$ & $\downarrow-$ \\
\hline \multicolumn{1}{l}{ Возраст >65 лет } & Другие причины & & \\
ХПН & $\downarrow$ & $\downarrow$ \\
Псевдогиперальдостеронизм 2 типа & - & $\downarrow$ \\
Беременность & - & $\uparrow \uparrow$ \\
Реноваскулярная АГ & $\uparrow$ & $\uparrow \uparrow$ \\
Злокачественная АГ & $\uparrow$ & $\uparrow \uparrow$
\end{tabular}

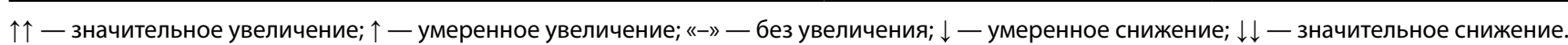


ложноотрицательные результаты тестирования у пациентов с легкой формой ПГА, ни один препарат не может вызывать ложноположительный результат при первичном тестировании, если использовать определенный cut-off для уровня альдостерона и ренина плазмы [48].

Длительное время исследование альдостерон-ренинового соотношения (АРС) считали самым надежным и доступным методом скрининга ПГА. Многочисленные научные работы продемонстрировали, что исследование АРС намного превзошло по чувствительности изолированные измерения калия или альдостерона, а по специфичности - ренина [49-51]. Однако АРС это тест с высокой вариабельностью, диагностическая чувствительность и специфичность которого варьируются от $66 \%$ до $100 \%$ и от $61 \%$ до $100 \%$ соответственно. Изменчивость показателей обусловлена влиянием целого ряда факторов: популяционной и индивидуальной вариабельности, различий в протоколах отбора проб, лабораторных исследованиях, единицах измерения, лекарственных препаратов [52,53].

Более того, при определении АРС отмечено большое количество ложноположительных результатов [54, 55]. По данным исследования Widimsky и соавт., повышение АРС при первичном тестировании было выявлено у $8 \%$ участников с АГ, подтверждение ПГА при дополнительном тестировании получено лишь у четверти [56].

Использование АРC может ввести в заблуждение, т.к. значение дроби в большой степени зависит от величины делителя. Например, при уровне альдостерона плазмы 3 нг/дл и активности ренина 0,1 нг/мл/ч соотношение будет равняться 30, что является ложноположительным значением. Очевидно, что при таком низком уровне альдостерона у пациента нет ПГА.

В связи с многочисленными дискордантными данными многие клиницисты отходят от тотального подсчета АРС всем пациентам с АГ, предпочитая производить дополнительную диагностику ПГА в основном в случаях с умеренной или высокой концентрацией альдостерона [57]. Соответственно, было предложено использовать определенный пороговый уровень альдостерона при подсчете АРС. Первоначально была предложена отметка в 15 нг/дл (410 пмоль/л) [58]. Однако в ряде работ было выявлено, что у $36 \%$ пациентов с впоследствии подтвержденной ПГА показатели альдостерона варьировали между 9 и 16 нг/дл (250-440 пмоль/л), при этом лишь у $4 \%$ данный показатель не достигал 10 нг/дл $[51,55,59]$.

С целью упрощения диагностики, снижения количества ложных результатов в клинической практике предлагается использовать абсолютные величины альдостерона и ренина.

Положительным первичным тестом на ПГА можно считать сочетание уровня альдостерона в верхней половине референса ( >10 нг/дл /100 пг/мл/277 пмоль/л) и активности ренина <1 нг/мл/ч (или прямого ренина ниже референсного значения) [60].

Уровень калия в сыворотке не является надежным ориентиром для скрининга ПГА, так как 72\% пациентов с ПГА имеют нормокалиемию $[60,61,62]$. Тем не менее целесообразна медикаментозная компенсация гипокалиемии, при ее наличии, до проведения дальнейших диагностических исследований или лечения.
- В связи с большим количеством ложноположительных результатов, крайне низкой вероятностью ПГА - не рекомендовано определение альдостерона и ренина плазмы у пациентов с отсутствием АГ (в т.ч. с инциденталомой надпочечников).

Уровень убедительности рекомендаций В (уровень достоверности доказательств 3).

Комментарии. Чтобы сократить медицинские расходы и ограничить последствия ложноположительных тестов, в рекомендациях эндокринного общества по ПГА рекомендуется тестировать только группы высокого риска на ПГА, обязательным критерием которого является наличие АГ. Более того, необоснованное обследование и лечение (например, прекращение приема антигипертензивных препаратов, тесты с солевой нагрузкой, инвазивные исследования, оперативное вмешательство) потенциально может причинить вред пациентам [60].

Существует ряд работ, оценивающих корреляцию APC, уровня альдостерона и ренина плазмы с несколькими сердечно-сосудистыми параметрами у нормотензивных пациентов и пациентов с АГ, никогда не принимавших лекарственные препараты, без клинических подозрений на ПГА и сопутствующих сердечно-сосудистых или почечных заболеваний. По результатам работ АРС был достоверно связан со скоростью пульсовой волны, без связи с другими гемодинамическими показателями. Взаимосвязи сывороточной концентрации как альдостерона, так и ренина и различий в гемодинамических переменных также не обнаружено [63-65].

- При получении отрицательного первичного теста на фоне некомпенсированной гипокалиемии и/или приема антигипертензивных препаратов, оказывающих возможное влияние на уровень ренина, рекомендовано повторное определение альдостерона, ренина на фоне отмены/модификации антигипертензивной терапии и компенсации гипокалиемии.

Уровень убедительности рекомендаций С (уровень достоверности доказательств 4).

Комментарии. Снижение уровня калия при высоком уровне альдостерона является физиологической реакцией организма [66]. В свою очередь, гипокалиемия снижает секрецию альдостерона, однако, это редко приводит к нормализации секреции альдостерона у пациентов с ПГА. В связи с чем для точности первичной диагностики рекомендуется нормализация показателей калия плазмы с последующим повторным определением уровня альдостерона, ренина [55].

Блокаторы кальциевых каналов и блокаторы a1-адренорецепторов в большинстве случаев не влияют на точность диагностики. Ингибиторы ангиотензинпревращающего фермента (АПФ) и блокаторы рецепторов ангиотензина (БРА) могут повышать уровень ренина у пациентов с легкой формой ПГА [55].

AМКР (например, спиронолактон и эплеренон) предотвращают активацию рецептора альдостероном, что приводит к потере натрия, уменьшению объема плазмы и повышению уровня ренина. Если показатели ренина плазмы или его активности не подавлены у пациента, получавшего АМКР, то необходимо повторное определение (альдостерона, ренина/ активности ренина, калия) через 6 нед на фоне отмены терапии. Однако наличие гипокалиемии, супрессии уровня ренина/активности 
ренина, несмотря на лечение АМКР, с высокой долей вероятности свидетельствует о неполной блокировке рецепторов минералокортикоидов и, как следствие, указывает на высокую вероятность наличия ПГА у такого пациента $[48,60]$.

С алгоритмом дальнейшей диагностики и лечения ПГА можно ознакомиться в соответствующих клинических рекомендациях.

\section{Гиперандрогения.}

- Не рекомендуется определять уровень половых гормонов и предшественников стероидных гормонов у пациентов с инциденталомами надпочечников, кроме случаев подозрения на АКР и/или наличия признаков гиперандрогении.

Уровень убедительности рекомендаций С (уровень достоверности доказательств 4).

Комментарий. Субклинические проявления других видов гормональной активности инциденталом, кроме вышеперечисленных вариантов, крайне редки, их определение актуально при обоснованном подозрении на АКР. При повышенной продукции половых гормонов клинические проявления достаточно яркие, в этом случае гормональное обследование надпочечников проводится, как правило, целенаправленно. При клиническом подозрении на изолированную или сочетанную гиперпродукцию стероидных гормонов рекомендовано определение: дегидроэпиандростерон-сульфата, андростендиона, тестостерона (у женщин), 17- $\beta$-эстрадиола (у мужчин и женщин в менопаузе) $[67,68]$. Наличие соответствующих КТ-признаков, сочетанной автономной гиперпродукции андрогенов и кортизола, предшественников стероидов или эстрадиола (у мужчин) с высокой долей вероятности свидетельствует об АКР $[69,70]$.

Исключением является неклассическая форма врожденной дисфункции коры надпочечников (ВДКН), которая может сопровождаться как односторонней, так и двусторонней надпочечниковой гиперплазией. Нет необходимости проводить пробу с АКТГ-стимуляцией и исследовать уровень предшественников кортизола - 17-гидроксипрогестерона (17-ОН прогестерон) и 11-дезоксикортизола всем пациентам с инциденталомами надпочечников. Исследование показано только при двусторонних опухолях надпочечников и наличии клинических проявлений гиперандрогении [71].

- Пациентам с двусторонними образованиями надпочечников рекомендована оценка клинических проявлений и гормональное обследование, идентичные таковым у пациентов с односторонними инциденталомами.

Уровень убедительности рекомендаций В (уровень достоверности доказательств 2).

Комментарий. Двусторонние образования надпочечников, которые выявляются в 10-15\% случаев, условно можно также разделить на гормонально-активные и гормонально-неактивные.

Двусторонняя гиперпродукция гормонов может приводить как к гиперкортицизму, гиперальдостеронизму, так и гиперкатехоламинемии. Лабораторная диагностика при данных формах ничем не отличается от таковой при одностороннем поражении [72].
Макронодулярная двусторонняя гиперплазия надпочечников (МДГН) является редкой причиной АКТГ-независимого гиперкортицизма. Она характеризуется двусторонними, заметно увеличенными надпочечниками в результате наличия множественных узлов, размер которых превышает 1 см. Данная патология не всегда сопровождается развитием манифестных форм СК, около половины пациентов имеют субклиническую форму [73].

Двусторонние альдостеронпродуцирующие аденомы выявляются крайне редко. Двустороннее поражение наиболее часто проявляется двусторонней диффузно-узелковой гиперплазией и выявляется примерно у 1/3 больных ПГА.

Двусторонний, мультифокальный характер поражения при ФХЦ/ПГ является индикатором наличия наследственного синдрома. По меньшей мере у трети пациентов причиной заболевания является наследственная мутация (NF1, RET, VHL, SDHD, SDHC, SDHB, EGLN1/PHD2, KIF1 $\beta$, SDH5/SDHAF2, IDH1, TMEM127, SDHA, MAX и HIF2a и др.). В связи с чем, помимо проведения лабораторной диагностики, для всех пациентов с ФХЦ/ПГ рекомендовано рассмотрение вопроса о выполнении генетического обследования [36].

- Пациентам с двусторонними образованиями надпочечников и отсутствием ФАПК рекомендуется определять уровень 17-гидроксипрогестерона с целью исключения ВДКН и выполнять диагностические тесты для исключения надпочечниковой недостаточности.

Уровень убедительности рекомендаций С (уровень достоверности доказательств 4).

Комментарий. Двустороннее поражение надпочечников может быть следствием ВДКН, в связи с чем рекомендуется проводить дополнительное обследование для исключения данного диагноза. Длительная декомпенсация надпочечниковой недостаточности ведет к гиперпластическим изменениям в надпочечниках, иногда приводящим к их значительному увеличению в объеме [71].

При адекватном неонатальном скрининге постановка диагноза осуществляется уже в раннем детском возрасте, это позволяет инициировать лечение максимально рано. Поэтому диагностика классических форм в более позднем возрасте, как правило, не требуется. Заподозрить классические формы ВДКН у взрослых можно при несоответствии паспортного и генетического пола, а также признаках хронической надпочечниковой недостаточности, которая будет проявляться мышечной слабостью, утомляемостью, потемнением кожных покровов в сочетании с симптомами гиперандрогении. При подозрении на НН рекомендовано определение уровня АКТГ, кортизола крови в утреннее время. При значительно повышенном уровне АКТГ (более 150 пг/мл) и уровне кортизола менее 500 нмоль/л диагноз первичной НН может быть установлен. При уровне АКТГ менее 150 пг/мл и уровне кортизола менее 500 нмоль/л требуется дополнительный стимуляционный тест (с синактеном короткого или пролонгированного действия) [74].

Для исключения ВДКН рекомендуется определение уровня 17-ОН-прогестерона в ранние утренние часы. Однако в некоторых случаях повышение уровня 17-ОН-прогестерона может указывать на повышенную секрецию предшественников стероидов, в частности, 
при злокачественных опухолях. В этих случаях рекомендовано определение уровня АКТГ в утренние часы, подавление которого исключает диагноз ВДКН $[75,76]$.

Диагностика неклассической формы 11ß-гидроксилазы осуществляется на основании повышения 11-дезоксикортизола. При получении показателей В «серой зоне» 17ОН-прогестерона (6-30 нмоль/л, или 2-10 нг/мл) и 11-дезоксикортизола, выявленных минимум при двукратном определении, проводится стимулирующий тест с синактеном (косинтропином, тетракозактидом - синтетическим аналогом АКТГ). Это является золотым стандартом диагностики неклассической формы ВДКН во всем мире. Однако на данный момент проведение данной пробы невозможно ввиду отсутствия регистрации короткого синактена [77].

При исключенной гормональной активности дифференциальную диагностику стоит проводить между двусторонними лимфомами, метастазами (рака иной локализации), миелолипомами, гематомами, реже причиной являются инфекционные и инфильтративные процессы, такие как туберкулез, гистоплазмоз, бластоцитоз, саркоидоз и амилоидоз [72].

\section{4. ИНСТРУМЕНТАЛЬНЫЕ ДИАГНОСТИЧЕСКИЕ ИССЛЕДОВАНИЯ.}

Определение злокачественного потенциала опухоли имеет ключевое значение в выборе тактики ведения. Крайне важно знать, является ли новообразование надпочечника злокачественным или доброкачественным, поскольку лечение зависит от установления этого факта, независимо от гормональной активности образования. Злокачественные образования могут потребовать срочного хирургического вмешательства и других методов лечения, а промедление может причинить вред.

MCКТ, МРТ и ПЭТ-КТ с ${ }^{18}$ ФДГ демонстрируют эффективность при диагностике образований надпочечников, однако наилучший метод лучевой диагностики до сих пор является предметом активных обсуждений. Рандомизированных исследований, сравнивающих различ-

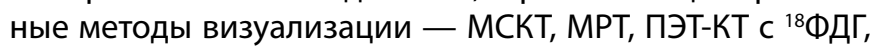
не проводилось. По данным результатов многочисленных исследований на достаточно маленьких выборках с большим доверительным интервалом (95\%), МСКТ имеет более высокую чувствительность (93-99\%), чем МРТ (81-99\%) [2, 78, 79, 80]. Дополнительными существенными преимуществами МСКТ являются возможность оценки критерия плотности опухоли в фазу вымывания (washout), лучшее пространственное разрешение при оценке инвазии в окружающие органы и ткани, диагностика опухолевых тромбов в венозную фазу исследования.

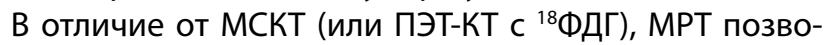
ляет избежать ионизирующего излучения и связанных с ним рисков для пациента. Однако количественная оценка интенсивности сигнала недостаточно стандартизирована между различными исследованиями, и, следовательно, доказательной базы для выполнения МРТ при диагностике злокачественных новообразований недостаточно, чтобы дать четкие рекомендации.

- Всем пациентам с инциденталомами надпочечников рекомендовано проведение визуализирующих исследований для выявления признаков доброка- чественности: гомогенность структуры, низкая плотность менее $10 \mathrm{HU}$ (т.е. признаки высокого содержания липидов). С этой целью исходно рекомендуется использование МСКТ без контрастного усиления.

Уровень убедительности рекомендаций А (уровень достоверности доказательств 2).

Комментарий. Низкий уровень нативной плотности по данным лучевых методов диагностики, в частности МСКТ, характерен для доброкачественных новообразований, к которым относятся новообразования из тканей надпочечников (гормонально-неактивные аденомы, альдостеромы, кортикостеромы), образования из неспецифичных для надпочечника тканей (липомы, миелолипомы, кисты, гематомы, абсцессы и др.).

Результаты 2 систематических обзоров и метаанализов подтвердили, что выявленная при МСКТ без контрастного усиления плотность ( $\leq 10 \mathrm{HU})$ во всех случаях соответствовала доброкачественному новообразованию, тогда как у пациентов с экстра-надпочечниковыми злокачественными новообразованиями только в 7\% метастазы имели плотность менее $10 \mathrm{HU}$ [79, 81, 82].

- Для диагностики злокачественного потенциала опухоли рекомендована оценка количественных денситометрических показателей при трехфазной КТ:

- плотность тканевого компонента до контрастирования (нативная);

- плотность в тканевую фазу контрастирования (артериальная и венозная фазы);

- плотность в отсроченную (через 10 мин после введения контраста) фазу контрастирования (фаза вымывания);

- наличие признаков инвазивного роста в соседние органы;

- наличие опухолевого или сосудистого тромбоза почечных вен, нижней полой вены.

Уровень убедительности рекомендаций В (уровень достоверности доказательств 2).

Комментарий. Для оценки КТ-фенотипа опухоли предложены различные варианты, наиболее удобный и часто используемый предложен M. Terzolo в 2011 г. и подтвержден результатами метаанализа.

- Доброкачественный КТ-фенотип: опухоль до 4 см, однородной структуры, низкой нативной плотности (менее 10-15 HU) - не требует 3-фазного КТ.

- Неопределенный КТ-фенотип: опухоли высокой плотности менее 4 см; опухоли низкой плотности более 4 см; опухоли мозаичной плотности (вследствие неоднородной структуры).

- Злокачественный КТ-фенотип: опухоль более 4 см высокой нативной КТ-плотности; любая опухоль с признаками инвазивного роста в соседние органы, опухолевого или сосудистого тромбоза $[13,83]$.

Необходимо обратить внимание, что существует различие между КТ-фенотипом и злокачественным потенциалом опухоли (последний является понятием клиническим и включает оценку множества факторов, в т.ч. КТ-фенотип). Именно на основании клинической оценки злокачественного потенциала и принимается клиническое решение.

Как было показано, АКР может быть выявлен у 1,9-4,2\% пациентов с инциденталомами надпочечников. Для оценки вероятности АКР и формирования 
показаний к оперативному вмешательству длительное время использовались 2 параметра: размер опухоли и скорость роста при динамическом наблюдении. Это приводило к большому числу необоснованных вмешательств при опухолях более 3-4 см и недооценке злокачественного потенциала образований малого размера при АКР или метастатических поражениях. Среди опухолей более 4 см четверть являлась злокачественными, при этом доля АКР при образованиях менее 6 см составила 10\%, а более 6 см - 20\% [84]. Оценка КТ-семиотики опухоли при образованиях 4 см и более имеет чувствительность 92-94\%.

В более поздних исследованиях, при введении денситометрических критериев злокачественности опухоли, удалось оптимизировать показания к оперативному лечению не только на основании размеров опухоли [79]. В настоящий момент основное внимание в диагностике АКР сконцентрировано на количественных показателях трехфазной МСКТ.

При исследовании КТ-плотности в разных фазах выведения контрастного вещества (wash-out) аденомы коры надпочечника значительно быстрее вымывают контрастное вещество, в то время как другие образования надпочечников, такие как метастазы и злокачественные новообразования, имеют тенденцию к задержке контрастного вещества.

КТ-семиотика инциденталом надпочечников оценивается в результате определения плотности жировых и нежировых структур в нативную фазу (жировые структуры имеют отрицательную плотность). Богатые липидами ткани характерны для доброкачественных аденом коры надпочечника, несмотря на это около 25\% доброкачественных аденом могут не иметь низкой нативной плотности [79].

Миелолипома надпочечников - относительно редкая опухоль, содержащая в основном жир и кроветворные элементы, напоминающие костный мозг. Миелолипомы обычно односторонние и гормонально-неактивные, могут достигать довольно больших размеров. Их легко отличить от аденом надпочечников по наличию обычно хорошо выраженного макроскопического жира на изображениях, они также могут содержать небольшие участки кальцификации [79]. В качестве подтверждающего метода возможно выполнение ультразвукового исследования (УЗИ). Ультразвуковыми признаками миелолипом являются гиперэхогенная, неоднородная структура и четкий контур образования [85].

Снижение КТ-плотности через 10 мин после введения контрастного вещества более чем на 50\% обладает высокой положительной прогностической значимостью (с высокими показателями чувствительности и специфичности - 100 и 98\% соответственно) в отношении диагностики доброкачественных аденом надпочечника.

Диагностика кистозных образований надпочечников при МСКТ затруднений, как правило, не вызывает. Истинные кисты описываются как образования с гомогенной структурой, четкими, ровными контурами и капсулой, в которой в отдельных случаях могут визуализироваться кальцинаты. Патогномоничным признаком является отсутствие накопления контрастного вещества в соответствующие фазы. Верификационным методом в данном случае, так же как и при миелолипомах, может быть УЗИ.
Эхографическими признаками кист/миелолипом будет являться анэхогенная, однородная структура с гиперэхогенным ободком [85]. Подобную кистам структуру при МСКТ имеют «старые» гематомы и гемангиомы.

Метастатическое поражение должно быть исключено/подтверждено в первую очередь у больных с анамнезом онкологического заболевания. Также вероятность метастатического поражения рассматривается при двустороннем поражении надпочечников, особенно при отсутствии явлений гормональной активности, при наличии КТ-признаков, характерных для метастазов [72]. Пациенты с подобными поражениями должны проходить онкологическое обследование для исключения первичного очага (в первую очередь рака почки, легкого, желудка, колоректального рака).

- При получении высокоплотных КТ-значений в нативную фазу, задержке контраста в отсроченной фазе рекомендовано оценивать КТ-фенотип опухоли как неопределенный (при размере до 4 см) или злокачественный (при большем размере).

Уровень убедительности рекомендаций В (уровень достоверности доказательств 2).

Комментарий. По данным систематического обзора и метаанализа, опухоли более 4 см с нативной плотностью более $20 \mathrm{HU}$ требуют дополнительных диагностических процедур. Результаты исследований свидетельствуют о том, что размер опухоли надпочечников более 4 см сам по себе не может быть индикатором злокачественности. Другим диагностическим фактором является плотность опухоли при МСКТ, которая в некоторых исследованиях вводится как лучшая характеристика для диагностики злокачественного новообразования. При ретроспективном анализе МСКТ-изображений было выявлено, что АКР обычно имеет большие размеры, неоднородную высокую плотность среднее значение нативной плотности составляло $34 \mathrm{HU}$ (диапазон 14-74). ROC-анализ показал, что пороговое значение $21 \mathrm{HU}$ обладает наилучшей диагностической точностью (чувствительность 96\%, специфичность 80\%) [83, 86].

- При выявлении образования надпочечника с неопределенным КТ-фенотипом и отсутствии гормональной гиперсекреции рекомендовано рассмотреть ситуацию в консилиуме, при этом возможны три варианта решений:

- проведение дополнительных визуализирующих исследований (ПЭТ-КТ с ФДГ);

- динамическое наблюдение с повторной КТ без контраста (или МРТ) через 3-6 мес;

- хирургическое лечение.

Уровень убедительности рекомендаций С (уровень достоверности доказательств 3).

Комментарий. Недавно ПЭТ с ${ }^{18}$ ФДГ была предложена как, возможно, лучший тест второй линии для оценки опухолей с неопределенном КТ-фенотипом $[87,88,89]$. По данным проведенного метаанализа, подтверждающего результаты

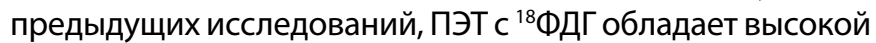
чувствительностью и специфичностью в диагностике злокачественных опухолей. В случае подозрения на АКР или метастазы рака иной локализации рекомендовано

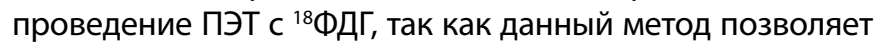
определить не только насыщенность опухолевых липидов, но и метаболическую активность образования, для измерения которой используется накопительный критерий SUV 
(standartised uptake value). По данным метаанализа, значение cut-off для SUVmax - 3, обладает чувствительностью 89\%, специфичностью 94\%, точностью 91\%, положительной прогностической ценностью 94\% и отрицательной прогностической ценностью 88\% [90].

Однако ПЭТ с ${ }^{18}$ ФДГ является дорогостоящей процедурой, которая ограничена возможностью проведения только в специализированных медицинских учреждениях. Необходимо принимать во внимание, что доброкачественные образования надпочечников также могут иметь повышенную метаболическую активность. Это в большей степени касается гормонально-активных аденом и ФХЦ [91, 92]. Во избежание получения ложноположительного результата рекомендовано перед направлением пациента на ПЭТ с ${ }^{18}$ ФДГ исключить гормональную активность образования надпочечника.

В качестве альтернативной тактики пациентам без серьезных подозрений на злокачественные новообразования, а также пожилым пациентам может быть предложено динамическое наблюдение. Контрольное обследование должно быть проведено через 6 мес после первичного исследования. Обоснованием является факт, что злокачественные новообразования надпочечников или метастазы могут увеличиваться в размерах за этот период времени, а отсутствие роста может быть расценено как индикатор доброкачественного процесса [2].

у пациентов с отягощенным онкологическим анамнезом и опухолью размерами более 3 см с плотностью более $20 \mathrm{HU}$ необходимо рассмотреть хирургическое лечение, в то время как для опухолей размерами менее 3 см с плотностью менее 20 HU может быть выбрана выжидательная тактика [83].

- В дифференциальном диагнозе органоспецифичных опухолей надпочечника пункционная биопсия не рекомендована, так как не имеет доказанных преимуществ, ассоциируется с низкой чувствительностью, специфичностью и высокой вероятностью осложнений

Уровень убедительности рекомендаций А (уровень достоверности доказательств 2).

Комментарий. Известный онкологический принцип необходимости морфологической предоперационной верификации опухоли в отношении опухолей надпочечников был поставлен под сомнение рядом исследований $[93,94]$. По данным систематического обзора и метаанализа, тотальное выполнение пункционной биопсии опухолей надпочечников не продемонстрировало улучшения результатов дифференциальной диагностики инциденталом, а напротив, привело к росту осложнений, ложноположительных и ложноотрицательных заключений [95]. При различных вариантах надпочечниковых опухолей диагностическая ценность предоперационной пункционной биопсии неудовлетворительна (чувствительность не превышает 65\%). Исключением является диагностика крайне редкой формы неходжкинской лимфомы с изолированным поражением надпочечников, для которой свойственны двусторонний инфильтративный характер поражения и быстрый темп роста опухоли. У пациентов нередко бывают косвенные признаки (выраженные явления общей интоксикации, повышение лактатдегидрогеназы в сыворотке крови и т.д.), позволяющие подозревать лимфому.
Попытки верификации диагноза ФХЦ с помощью цитологического исследования датируются периодом, когда отсутствовала возможность качественной диагностики опухолевой гиперкатехоламинемии. В настоящий момент пункционная биопсия для диагностики ФХЦ не рекомендована [33]. При биопсии хромаффинных опухолей в кровяное русло высвобождается большое количество катехоламинов, что может привести к развитию высокоамплитудных гипертензивных кризов, отека легких, фатальных аритмий, синдрома «неуправляемой гемодинамики» и внезапной сердечной смерти.

Мы категорически не рекомендуем проведение биопсии надпочечников, если есть вероятность, что данное образование является АКР, так как в данном случае биопсия сопряжена с риском распространения опухоли, резко снижающим вероятность резекции R0 и, как следствие, значительно ухудшающим прогноз. Единственным исключением может быть формальное подтверждение диагноза неоперабельной опухоли для подбора соответствующей противоопухолевой лекарственной терапии.

- Пункционная биопсия опухоли надпочечника рекомендована при обоснованном анамнестическом подозрении на его метастатическое поражение при одновременном соблюдении 3 условий:

1. образование является гормонально-неактивным (ФХЦ должна быть исключена в первую очередь);

2. КТ-фенотип не соответствует доброкачественному;

3. результаты морфологического исследования могут отразиться на тактике лечения.

Уровень убедительности рекомендаций С (уровень достоверности доказательств 4).

Комментарий. Биопсия надпочечников сопряжена со значительным процедурным риском. Биопсия рекомендуется только для новообразований, имеющих злокачественный КТ-фенотип, в случае, когда результат может повлиять на решение о клиническом лечении.

По данным метаанализа, биопсия надпочечников имеет $87 \%$ чувствительность и $100 \%$ специфичность для общей диагностики злокачественных новообразований. Аналогичная эффективность была отмечена для диагностики метастазов (чувствительность $87 \%$ и специфичность 96\%). Более низкая эффективность биопсии надпочечников при диагностике карциномы коры надпочечников (чувствительность $70 \%$ и специфичность 96\%). Кроме того, при проведении биопсии высока вероятность получения недостаточного количества тканевого материала и неэффективности последующей оценки по системе баллов Weiss, которая обычно используется для дифференциальной диагностики доброкачественных и злокачественных образований надпочечников и поэтому не рекомендуется в этой ситуации [95].

Случаи, в которых применение биопсии обосновано, указаны ранее в пункте 2.4.5.

- При двусторонних образованиях надпочечников рекомендуется оценивать каждое образование в соответствии с правилами обследования при односторонних инциденталомах с целью установления их потенциальной злокачественности/доброкачественности.

Уровень убедительности рекомендаций А (уровень достоверности доказательств 1). 
Комментарий. Двусторонние образования надпочечников выявляются в 10-15\% случаев, причинами которых могу быть метастатические поражения, двусторонние ФХЦ, МДГН, ВДКН, двусторонние аденомы, миелолипомы, лимфомы и др. [79].

Более того, двусторонние образования надпочечников могут представлять собой одновременное возникновение различных патологий, таких как аденома, ФХЦ, киста, миелолипома и др. В систематических обзорах и метаанализах исследовались случаи как с односторонним, так и с двусторонним поражением, следовательно, каждую опухоль необходимо оценить в соответствии с рекомендациями (п. 2.4) [23, 26, 82, 83, 90, 95].

\section{3. ЛЕЧЕНИЕ, ВКЛЮЧАЯ МЕДИКАМЕНТОЗНУЮ \\ И НЕМЕДИКАМЕНТОЗНУЮ ТЕРАПИЮ, ДИЕТОТЕРАПИЮ, ОБЕЗБОЛИВАНИЕ, МЕДИЦИНСКИЕ ПОКАЗАНИЯ И ПРОТИВОПОКАЗАНИЯ К ПРИМЕНЕНИЮ МЕТОДОВ ЛЕЧЕНИЯ}

\section{1. ХИРУРГИЧЕСКОЕ ЛЕЧЕНИЕ.}

Решение о проведении оперативного вмешательства пациентам с инциденталомой надпочечника должно приниматься индивидуально. Сложные случаи по возможности должны разбираться на консилиумах специалистов разных направлений.

- Рекомендовано проведение адреналэктомии в качестве стандарта лечения односторонних опухолей надпочечников с клинически значимым избытком гормонов.

Уровень убедительности рекомендаций А (уровень достоверности доказательств 2).

- При оценке показаний к хирургическому лечению у пациентов с доброкачественной аденомой надпочечников, ФАПК и заболеваниями, потенциально связанными с гиперсекрецией кортизола, предлагается использовать индивидуальный подход. Должны учитываться возраст, уровень гиперсекреции, сопутствующие заболевания, риск операции и анестезии и желание пациента.

Уровень убедительности рекомендаций D (уровень достоверности доказательств 4).

Комментарий. В соответствии с клиническими рекомендациями существует консенсус в отношении того, что опухоли надпочечников, приводящие к клинически значимому избытку гормонов (например, СК, ПГА или ФХ/ПГ), требуют хирургического лечения [21, 33, 60]. Для этих опухолей должны применяться те же правила в отношении хирургического доступа, что и для ГНО.

Показания к оперативному лечению СК:

\section{- манифестная форма.}

При односторонних процессах эффективность адреналэктомии достигает почти $100 \%$ в лечении гиперкортицизма, ассоциированного с высоким риском развития тяжелых сердечно-сосудистых осложнений, инвалидизации и преждевременной смерти. При МДГН рекомендовано проведение односторонней адреналэктомии со стороны большего поражения, с последующей оценкой гормонального статуса для определения тактики ведения [21].
Показания к операции, опирающиеся исключительно на диагноз ФАПК при доброкачественных опухолях надпочечника, активно дискутируются. В настоящий момент на основании опубликованных исследований определенности не достигнуто.

Высокая распространенность в популяции, особенно с увеличением возраста, таких коморбидных состояний, как АГ, остеопороз, СД, ожирение, в генезе которых может быть первопричиной гиперкортицизм, делает крайне затруднительным определение происхождения этих патологий при наличии инциденталомы с доказанной ФАПК. Индивидуальный подход имеет принципиальное значение для определения тактики ведения таких пациентов. «ЗА» операцию - молодой возраст больного, возможное отрицательное влияние ФАПК на вышеперечисленную коморбидность. «ПРОТИВ» - пожилой возраст, высокий риск оперативного лечения, отсутствие заболеваний, в генезе которых может участвовать гиперкортицизм [96].

Показания к оперативному лечению ПГА:

- односторонняя гиперпродукция альдостерона (альдостеронпродуцирующей аденомой).

Одностороннее эндоскопическое удаление надпочечника улучшает течение АГ, нивелирует гипокалиемию почти у $100 \%$ пациентов с односторонними вариантами ПГА. Клиническое улучшение - нормализация/улучшение показателей АД в большинстве случаев наступает через 1-6 мес после односторонней адреналэктомии при альдостеронпродуцирующей альдостероме [33].

\section{Показания к оперативному лечению ФХ/ПГ.}

Пациентам с надпочечниковой ФХЦ рекомендуется проводить эндоскопическую адреналэктомию. Для больших (более 8/10 см) и инвазивных опухолей предпочтителен открытый доступ во избежание повреждения капсулы опухоли и ее диссеминирования. Эндоскопическое удаление вненадпочечниковых ФХЦ/ПГ рекомендуется только в специализированных ведущих хирургических центрах, исключением являются неинвазивные параганглиомы малого размера [60]. Также всем пациентам с ФХЦ/ПГ рекомендована предоперационная подготовка $\alpha-$ и $\beta$-адреноблокаторами, с последующей оценкой эффективности проводимой терапии (коррекция гиповолемического синдрома) непосредственно перед оперативным вмешательством.

- Оперативное вмешательство при бессимптомных образованиях надпочечников с очевидными признаками доброкачественности по данным визуализирующих методов (в том числе с лабораторно доказанной ФАПК) не рекомендуется.

Уровень убедительности рекомендаций А (уровень достоверности доказательств 2).

Комментарий. Большинство инциденталом надпочечников - это нефункционирующие доброкачественные образования (например, аденомы, миелолипомы), которые не представляют опасности и в большинстве случаев не требуют хирургического вмешательства. Исключением являются объемные доброкачественные образования значительных размеров, компримирующие окружающие структуры.

Немногочисленные литературные данные, касающиеся длительного наблюдения пациентов (период 
наблюдения 2-5 лет) с доброкачественными ГНО надпочечников, показывают, что в 5-25\% случаев отмечается тенденция к увеличению опухолей в размере, при этом лишь в 9\% случаев рост составил более 1 см [23, 32, 97, 98]. Опасность озлокачествления стремится к нулю. По данным метаанализа, среди 32 исследований, включающих 4121 пациента с доброкачественными критериями ГНО и ФАПК, ни у одного из них в последующем не развился АКР [23].

Адреналэктомия при бессимптомной ФАПК не несет в себе клинической значимости, при этом возрастает вероятность ухудшения качества жизни: риски операции и анестезии, послеоперационная надпочечниковая недостаточность и необходимость ее коррекции.

- При выявлении гормонально-неактивного образования надпочечника со злокачественным КТ-фенотипом рекомендовано оперативное лечение в наиболее короткие сроки после постановки диагноза. В качестве предоперационного обследования предлагаются проведение ПЭТ-КТ с ${ }^{18}$ ФДГ для стадирования процесса и исследование уровней половых гормонов и их стероидных предшественников.

Уровень убедительности рекомендаций А (уровень достоверности доказательств 2).

Комментарий. В случае наличия в анамнезе онкологического заболевания и высокой вероятности метастатического поражения, когда образование надпочечников является потенциально единственным метастазом и его удаление представляется разумным с онкологической точки зрения (способствует улучшению качества/продолжительности жизни), следует рассмотреть возможность хирургического вмешательства.

Единственным возможным вариантом полного излечения при АКР в настоящее время является полное хирургическое удаление опухоли в кратчайшие сроки после ее обнаружения. Акцент на срочности оперативного вмешательства связан с быстрым увеличением размеров опухоли и высокой вероятностью метастазирования. Отмечается значительное снижение 5- и 10-летней выживаемости с каждой последующей стадией. Если на момент обнаружения опухоли метастазы не выявляются, то вероятность ремиссии заболевания, безусловно, выше [10].

Существуют немногочисленные нерандомизированные когортные исследования, а также систематический обзор с метаанализом по хирургическому лечению АКР, сравнивающие эндоскопическую и открытую адреналэктомию. По результатам вышеуказанных работ, эндоскопическая операция по поводу локализованного АКР безопасна и эффективна, если ее проводят опытные хирурги в специализированных центрах в мультидисциплинарных командах. Пациентам с местнораспространенным процессом, большими опухолями (более $8 \mathrm{~cm}$ ) рекомендуется проводить открытую операцию с целью снижения риска повреждения капсулы и диссеминирования процесса [99-108],

Ввиду низкой специфичности и положительной прогностической ценности остается дискутабельным вопрос о проведении ПЭТ с ${ }^{18}$ ФДГ всем пациентам с подозрением на злокачественное образование надпочечника. Несомненным преимуществом ПЭТ с ${ }^{18}$ ФДГ является визуализация всего тела, в особенности за пределами грудной клетки и брюшной полости, особенно для отдаленных ме-

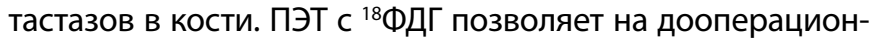

ном этапе определить распространенность опухолевого процесса. В качестве альтернативы возможно использование МСКТ органов грудной клетки, брюшной полости и малого таза с контрастным усилением $[10,90]$.

Исследование гормональной активности опухоли надпочечника строго регламентировано, и его результаты должны учитываться для планирования предоперационной подготовки и последующего ведения пациента (см. п. 2.3; 3.1.5).

- Периоперационное назначение глюкокортикоидов рекомендуется всем пациентам при хирургических вмешательствах по поводу опухолей надпочечников с доказанной ФАПК.

Уровень убедительности рекомендаций В (уровень достоверности доказательств 3).

Комментарий. Длительное течение ФАПК может привести к жизнеугрожающему состоянию (острой надпочечниковой недостаточности) после удаления источника избытка кортизола, это обусловлено подавлением выработки АКТГ и атрофией контралатерального надпочечника. По данным систематического обзора Dalmazi и соавт., в послеоперационном периоде у всех пациентов с манифестным СК и у 50\% пациентов с ФАПК развивается НH $[29,109]$.

Рекомендуется пери- и послеоперационная заместительная терапия глюкокортикоидами (предпочтительно гидрокортизоном) у пациентов с опухолью надпочечников с ФАПК даже при отсутствии клинических признаков избытка кортизола. В послеоперационном периоде доза глюкокортикоидов должна быть снижена индивидуально. Длительность заместительной терапии также индивидуальна, как правило, пациенты возвращаются в состояние эукортицизма в течение года после операции [21, 31].

- При двусторонних инциденталомах рекомендуется использовать аналогичные показания к оперативному лечению и принципы динамического наблюдения, что и при односторонних образованиях.

Уровень убедительности рекомендаций С (уровень достоверности доказательств 4).

Комментарии. Двусторонняя адреналэктомия ассоциирована с высоким риском смерти вследствие кризов надпочечниковой недостаточности, снижением качества жизни, пожизненной заместительной терапией, трудностью подбора оптимальных доз гормональных препаратов. При двусторонней ФХЦ данный вид лечения единственно эффективный, в отличие от двусторонней формы ПГА, где рекомендовано консервативное ведение пациентов (лечение препаратами АМКР) [33, 60].

Двустороння адреналэктомия как единственный метод лечения МДГН была поставлена под сомнение в 2015 г., когда Debillion и соавт. опубликовали результаты по выполнению односторонней адреналэктомии у пациентов с манифестным гиперкортицизмом, вызванным МДГН. В ходе 5-летнего периода наблюдения у всех прооперированных пациентов наблюдалась стойкая ремиссия, что свидетельствовало о высокой эффективности односторонней операции [110]. Впоследствии данный результат был подтвержден в многочисленных работах [111-113]. При возникновении рецидива заболевания рекомендуется рассмотрение повторного хирургического вмешательства или терапевтического лечения, если оно представляется возможным. 
4. МЕДИЦИНСКАЯ РЕАБИЛИТАЦИЯ И САНАТОРНОКУРОРТНОЕ ЛЕЧЕНИЕ, МЕДИЦИНСКИЕ ПОКАЗАНИЯ И ПРОТИВОПОКАЗАНИЯ К ПРИМЕНЕНИЮ МЕТОДОВ РЕАБИЛИТАЦИИ, В ТОМ ЧИСЛЕ ОСНОВАННЫХ НА ИСПОЛЬЗОВАНИИ ПРИРОДНЫХ ЛЕЧЕБНЫХ ФАКТОРОВ.

Специфических реабилитационных мероприятий в отношении пациентов с инциденталомами надпочечника не разработано.

\section{5. ПРОФИЛАКТИКА И ДИСПАНСЕРНОЕ НАБЛЮДЕНИЕ, МЕДИЦИНСКИЕ ПОКАЗАНИЯ И ПРОТИВОПОКАЗАНИЯ К ПРИМЕНЕНИЮ МЕТОДОВ ПРОФИЛАКТИКИ.}

\section{Динамическое наблюдение.}

Параметры динамического наблюдения пациентов с инциденталомами являются предметом постоянных дискуссий. Имеющиеся данные о динамическом наблюдении за пациентами с инциденталомами надпочечников позволяют предположить, что подавляющее большинство опухолей надпочечников, классифицированных как доброкачественные при постановке диагноза, остаются стабильными с течением времени [9, 23, 32, 114-116].

По данным Young. и соавт., при последовательном наблюдении за больными с различными вариантами надпочечниковых опухолей, рост корковой аденомы и ФХЦ составляет 0,5-1,0 см в год, в то время как для АКР характерен быстрый темп роста (>2 см ежегодно) [8]. Примерно в 4\% случаев сообщалось о периодическом уменьшении размеров или даже полном исчезновении новообразования надпочечников, чаще всего ими являлись кисты, гематомы, псевдонадпочечниковые образования [13, 114, 117].

- Проведение динамических визуализирующих исследований пациентам с образованиями менее 4 см и доброкачественным КТ-фенотипом при отсутствии роста более 5 мм за первый год наблюдения не рекомендовано.

Уровень убедительности рекомендаций А (уровень достоверности доказательств 2).

Комментарий. Среди более чем 2300 пациентов, включенных в исследования по критериям отсутствия гормональной активности и низкого злокачественного потенциала, за 2 года наблюдения не было пропущено ни одного случая AKP $[9,13]$. В результатах метаанализа Elhassan и соавт. средняя продолжительность наблюдения составила чуть более 4 лет, за данный период лишь в 2,5\% случаев инциденталом зафиксирован рост более 1 см [23]. По этой причине проведение повторных радиологических исследований при явной доброкачественности процесса нецелесообразно.

Berland и соавт. в своем обзоре указывают на отсутствие необходимости в последующем наблюдении, если новообразование надпочечника было квалифицировано как миелолипома или киста, а также подтверждают, что отсутствие быстрого роста новообразования надпочечника в течение 1 года или более делает весьма вероятным доброкачественный диагноз [118].

- При образованиях с неопределенным КТ-фенотипом с целью динамической оценки размеров опухоли (если на основании первичной диагностики было выбрано наблюдение) рекомендована повторная КТ/МРТ через 3-6 мес. Хирургическое лечение показано при увеличении размеров образования за короткий период наблюдения на 20\% (или увеличение максимального диаметра более 5 мм).

Уровень убедительности рекомендаций С (уровень достоверности доказательств 4).

Комментарий. Соответственно, для тех опухолей, доброкачественная природа которых не может быть определена с уверенностью при первичном обследовании, рекомендовано повторное обследование с целью раннего распознавания роста образования $[4,69]$.

Отсутствие роста в течение 12 мес делает маловероятным наличие злокачественной опухоли, в случае ее быстрого прогрессирования рекомендовано оперативное лечение.

Общепринятых критериев роста образования надпочечника не существует. Fassnacht и соавт. предложена адаптация части критериев прогрессирования заболевания из системы RECIST - увеличение суммы наибольших размеров не менее чем на 20\%, абсолютное увеличение размеров не менее чем на 5 мм [119].

- При первичной диагностике, исключившей гормональную гиперсекрецию, повторное гормональное исследование не рекомендовано. Повторные исследования гормонов рекомендованы при манифестации новых клинических симптомов, потенциально связанных с гормональной гиперпродукцией, либо если происходит необъяснимая декомпенсация коморбидных патологий (АГ, сахарного диабета 2 типа, ожирения).

Уровень убедительности рекомендаций С (уровень достоверности доказательств 4).

Комментарий. Ранее пятилетний риск возникновения гиперсекреции гормонов у первоначально гормонально-неактивного образования оценивался примерно в 0,3\%, однако, по данным последних исследований, этот риск снизился до 0,1\%. В связи с этим систематические последующие гормональные исследования в данном случае не рекомендованы $[9,13]$.

По результатам систематического анализа с применением мета-анализа, развитие ФАПК без признаков манифестного гиперкортицизма может произойти у 8-14\% пациентов с ГНО. Несмотря на этот факт, ежегодная гормональная оценка сопряжена с высоким риском ложноположительных результатов, в связи с чем первостепенно рекомендована оценка коморбидных состояний - АГ, СД, ожирения, и только в случае декомпенсации вышеуказанных состояний целесообразно повторное гормональное обследование [120].

- Пациентам с образованием надпочечника и ФАПК бессимптомного течения или же тем, которым в силу каких-то обстоятельств оперативное лечение не проводилось, рекомендован ежегодный мониторинг сопутствующих состояний, потенциально связанных с его гиперсекрецией. На основании полученных данных в динамике может обсуждаться целесообразность хирургического лечения.

Уровень убедительности рекомендаций С (уровень достоверности доказательств 4).

Комментарий. Как обсуждалось выше, ФАПК тесно связана с рядом коморбидных состояний, таких как АГ, 
остеопороз, СД, ожирение, в генезе которых гиперкортицизм может иметь значительное влияние.

Наблюдение пациентов с ФАПК должно включать оценку сопутствующих заболеваний, потенциально связанных с избытком кортизола. При возникновении или ухудшении этих коморбидных патологий необходимо повторное гормональное обследование с последующим решением о необходимости проведения оперативного лечения [23].

\section{6. ОРГАНИЗАЦИЯ ОКАЗАНИЯ МЕДИЦИНСКОЙ помощи.}

Амбулаторный/госпитальный этап оказания медичинской помощи:

- проведение лабораторного обследования - исключение/выявление гормональной активности;

- проведение инструментальной диагностики (МСКТ/

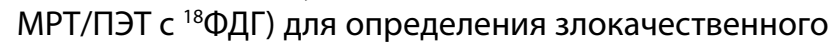
потенциала опухоли;

- консультации смежных специалистов по показаниям;
- коррекция сопутствующих коморбидных состояний на дооперационном/послеоперационном ведении.

Как следует из определения, инциденталома - это собирательный феномен, требующий нозологической конкретизации. Диагноз «инциденталома» выставляется до момента постановки окончательного диагноза, который определяется результатами соответствующих лабораторных и инструментальных исследований.

Соответственно, показания для госпитализации, выписки и нормативные документы представлены в клинических рекомендациях соответствующих нозологий.

\section{7. ДОПОЛНИТЕЛЬНАЯ ИНФОРМАЦИЯ \\ (В ТОМ ЧИСЛЕ ФАКТОРЫ, ВЛИЯЮЩИЕ НА ИСХОД ЗАБОЛЕВАНИЯ ИЛИ СОСТОЯНИЯ)}

\section{Факторы, влияющие на исход заболевания:}

1. своевременность постановки окончательного диагноза и проведенного лечения;

2. коррекция коморбидных состояний.

\section{КРИТЕРИИ ОЦЕНКИ КАЧЕСТВА МЕДИЦИНСКОЙ ПОМОЩИ}

\begin{tabular}{|c|c|c|}
\hline № & Критерии качества & $\begin{array}{c}\text { Оценка } \\
\text { выполнения } \\
\text { (Да/Нет) }\end{array}$ \\
\hline 1 & $\begin{array}{l}\text { Выполнено определение кортизола в ранние утренние часы на фоне подавляющего } \\
\text { теста с } 1 \text { мг дексаметазона. При отсутствии физиологического подавления уровня } \\
\text { кортизола в качестве подтверждающего теста выполнено определение АКТГ } \\
\text { в утренние часы }\end{array}$ & Да/Нет \\
\hline 2 & $\begin{array}{l}\text { Выполнено определение уровней метанефрина и норметанефрина в суточной моче } \\
\text { или плазме }\end{array}$ & Да/Нет \\
\hline 3 & $\begin{array}{l}\text { Выполнено определение уровней альдостерона, ренина и калия плазмы для } \\
\text { исключения первичного гиперальдостеронизма при наличии у пациента с опухолью } \\
\text { надпочечника артериальной гипертензии }\end{array}$ & Да/Нет \\
\hline 4 & $\begin{array}{l}\text { Выполнено определение стероидных гормонов сыворотки крови: } \\
\text { дегидроэпиандростерон-сульфат, 17-оксипрогестерон, андростендион, тестостерон } \\
\text { (у женщин), 17-ß-эстрадиол (у мужчин и женщин в менопаузе), 11-дезоксикортизол } \\
\text { при клиническом подозрении на изолированную или сочетанную (с } \\
\text { гиперкортицизмом) опухолевую гиперпродукцию половых гормонов при подозрении } \\
\text { на АКР и признаках гиперандрогении }\end{array}$ & Да/Нет \\
\hline
\end{tabular}

Выполнено МСКТ без контрастного усиления. При сомнительной КТ-семиотике выполнено МСКТ с контрастным усилением органов брюшной полости, определены

5 плотность тканевого компонента до контрастирования (нативная), плотность в тканевую фазу контрастирования (артериальная и венозная фазы), плотность Да/Нет в отсроченную (через 10 мин после введения контраста) фазу контрастирования (фаза вымывания)

6 Выполнено ${ }^{18}$ ФДГ-ПЭТ/КТ, оценка динамики роста по МСКТ как один из параметров клинической верификации злокачественного потенциала

Да/Нет

7 Выбран и выполнен оптимальный объем оперативного вмешательства Да/Нет

8 При наличии гиперкортицизма в послеоперационном периоде проведена заместительная терапия надпочечниковой недостаточности

Да/Нет

Всем пациентам на основании результатов динамического наблюдения и обследования проводится необходимая коррекция лечения и плана дальнейшего ведения 


\section{ДОПОЛНИТЕЛЬНАЯ ИНФОРМАЦИЯ}

Порядок обновления клинических рекомендаций. Механизм обновления клинических рекомендаций предусматривает их систематическую актуализацию - не реже чем 1 раз в 3 года, а также при появлении новых данных с позиции доказательной медицины по вопросам диагностики, лечения, профилактики и реабилитации конкретных заболеваний, наличии обоснованных дополнений/замечаний к ранее утвержденным КР, но не чаще 1 раза в 6 мес.

Источники финансирования. Работа выполнена по инициативе авторов без привлечения финансирования.
Конфликт интересов. Авторы декларируют отсутствие явных и потенциальных конфликтов интересов, связанных с содержанием настоящей статьи.

Участие авторов. Все авторы одобрили финальную версию статьи перед публикацией, выразили согласие нести ответственность за все аспекты работы, подразумевающую надлежащее изучение и решение вопросов, связанных с точностью или добросовестностью любой части работы.

Конфликт интересов. Авторы декларируют отсутствие явных и потенциальных конфликтов интересов, связанных с публикацией настоящей статьи.

\section{СПИСОК ЛИТЕРАТУРЫ | REFERENCES}

1. Бельцевич Д.Г., Кузнецов Н.С., Солдатова Т.В., Ванушко В.Э. Инциденталома надпочечников // Эндокринная хирургия. - 2009. T. 4. - №1. - C. 19-23. [Beltsevich DG, Kuznetsov NS, Soldatova TV, Vanushko VE. Adrenal incidentaloma. Endocrine Surgery. 2009;3(1):19-23. (In Russ.)]. doi: https://doi.org/10.14341/2306-3513-2009-1-19-23

2. Fassnacht M, Arlt W, Bancos I, et al. Management of adrenal incidentalomas: European Society of Endocrinology Clinical Practice Guideline in collaboration with the European Network for the Study of Adrenal Tumors. Eur J Endocrinol. 2016;175(2):G1-G34. doi: https://doi.org/10.1530/EJE-16-0467

3. Mansmann G, Lau J, Balk E, et al. The clinically inapparent adrenal mass: update in diagnosis and management. Endocr Rev. 2004;25(2):309-340. doi: https://doi.org/10.1210/er.2002-0031

4. Berruti A, Baudin E, Gelderblom H, et al. Adrenal cancer: ESMO Clinical Practice Guidelines for diagnosis, treatment and follow-up. Ann Oncol. 2012;23 Suppl 7:vii131-vii138. doi: https://doi.org/10.1093/annonc/mds231

5. Clark OH, Benson AB 3rd, Berlin JD, et al. NCCN Clinical Practice Guidelines in Oncology: neuroendocrine tumors. J Nat/ Compr Canc Netw. 2009;7(7):712-747. doi: https://doi.org/10.6004/jnccn.2009.0050

6. Kloos RT, Gross MD, Francis IR, et al. Incidentally discovered adrenal masses. Endocr Rev. 1995;16(4):460-484.

doi: https://doi.org/10.1210/edrv-16-4-460

7. Bovio S, Cataldi A, Reimondo G, et al. Prevalence of adrenal incidentaloma in a contemporary computerized tomography series. J Endocrinol Invest. 2006;29(4):298-302. doi: https://doi.org/10.1007/BF03344099

8. Young WF Jr. Clinical practice. The incidentally discovered adrenal mass. N Engl J Med. 2007;356(6):601-610. doi: https://doi.org/10.1056/NEJMcp065470

9. Cawood TJ, Hunt PJ, O'Shea D, et al. Recommended evaluation of adrenal incidentalomas is costly, has high false-positive rates and confers a risk of fatal cancer that is similar to the risk of the adrenal lesion becoming malignant; time for a rethink?. Eur J Endocrinol. 2009;161(4):513-527. doi: https://doi.org/10.1530/EJE-09-0234

10. Fassnacht M, Dekkers OM, Else T, et al. European Society of Endocrinology Clinical Practice Guidelines on the management of adrenocortical carcinoma in adults, in collaboration with the European Network for the Study of Adrenal Tumors. Eur J Endocrinol. 2018;179(4):G1-G46. Published 2018 Oct 1. doi: https://doi.org/10.1530/EJE-18-0608

11. Ahmed AA, Thomas AJ, Ganeshan DM, et al. Adrenal cortical carcinoma: pathology, genomics, prognosis, imaging features, and mimics with impact on management. Abdom Radiol (NY). 2020;45(4):945-963. doi: https://doi.org/10.1007/s00261-019-02371-y

12. Gross MD, Shapiro B. Clinical review 50: Clinically silent adrenal masses. J Clin Endocrinol Metab. 1993;77(4):885-888. doi: https://doi.org/10.1210/jcem.77.4.8408461

13. Terzolo M, Stigliano A, Chiodini I, et al. AME position statement on adrenal incidentaloma. Eur J Endocrinol. 2011;164(6):851-870. doi: https://doi.org/10.1530/EJE-10-1147

14. Terzolo M, Bovio S, Reimondo G, et al. Subclinical Cushing's syndrome in adrenal incidentalomas. Endocrinol Metab Clin North Am. 2005;34(2):423-x. doi: https://doi.org/10.1016/j.ecl.2005.01.008

15. Terzolo M, Bovio S, Pia A, et al. Midnight serum cortisol as a marker of increased cardiovascular risk in patients with a clinically inapparent adrenal adenoma. Eur J Endocrinol. 2005;153(2):307-315. doi: https://doi.org/10.1530/eje.1.01959
16. Tauchmanovà $L$, Rossi $R$, Biondi $B$, et al. Patients with subclinical Cushing's syndrome due to adrenal adenoma have increased cardiovascular risk. J Clin Endocrinol Metab. 2002;87(11):4872-4878. doi: https://doi.org/10.1210/jc.2001-011766

17. Morelli V, Masserini B, Salcuni AS, et al. Subclinical hypercortisolism: correlation between biochemical diagnostic criteria and clinical aspects. Clin Endocrinol (Oxf). 2010;73(2):161-166 doi: https://doi.org/10.1111/j.1365-2265.2010.03794.x

18. Mantero F, Terzolo M, Arnaldi G, et al. A survey on adrenal incidentaloma in Italy. Study Group on Adrenal Tumors of the Italian Society of Endocrinology. J Clin Endocrinol Metab. 2000;85(2):637-644. doi: https://doi.org/10.1210/jcem.85.2.6372

19. Kirkby-Bott J, Brunaud L, Mathonet M, et al. Ectopic hormone-secreting pheochromocytoma: a francophone observational study. World J Surg. 2012;36(6):1382-1388. doi: https://doi.org/10.1007/s00268-012-1488-1

20. Мельниченко Г.А., Алексеев Б.Я., Бельцевич Д.Г., и др. Клинические рекомендации. Рак коры надпочечников (адренокортикальный рак). 2020. [Melnichenko GA, Aleskeev BY, Beltsevich DG, et al. Clinical practice guidelines. Adrenal cortex cancer (adrenocortical cancer). 2020. (In Russ.)].

21. Nieman LK, Biller BM, Findling JW, et al. Treatment of Cushing's Syndrome: An Endocrine Society Clinical Practice Guideline. J Clin Endocrinol Metab. 2015;100(8):2807-2831. doi: https://doi.org/10.1210/jc.2015-1818

22. Libè R, Dall'Asta C, Barbetta L, et al. Long-term follow-up study of patients with adrenal incidentalomas. Eur J Endocrinol. 2002;147(4):489-494. doi: https://doi.org/10.1530/eje.0.1470489

23. Elhassan YS, Alahdab F, Prete A, et al. Natural History of Adrenal Incidentalomas With and Without Mild Autonomous Cortisol Excess: A Systematic Review and Meta-analysis. Ann Intern Med. 2019;171(2):107-116. doi: https://doi.org/10.7326/M18-3630

24. Кузнецов Н.С., Бельцевич Д.Г., Ванушко В.Э., и др. Дифференциальная диагностика инциденталом надпочечников // Эндокринная хирургия. - 2011. - T. 5. - №1. - C.5-16. [Kuznetsov NS, Beltsevich DG, Vanushko VE, et al. Differential diagnostics of adrenal incedentaloma. Endocrine Surgery. 2011;5(1):5-16. (In Russ.)]. doi: https://doi.org/10.14341/2306-3513-2011-1-5-16

25. Nieman LK, Biller BM, Findling JW, et al. The diagnosis of Cushing's syndrome: an Endocrine Society Clinical Practice Guideline. J Clin Endocrinol Metab. 2008;93(5):1526-1540. doi: https://doi.org/10.1210/jc.2008-0125

26. Ceccato F, Antonelli G, Frigo AC, et al. First-line screening tests for Cushing's syndrome in patients with adrenal incidentaloma: the role of urinary free cortisol measured by LC-MS/MS. J Endocrinol Invest. 2017:40(7):753-760. doi: https://doi.org/10.1007/s40618-017-0644-8

27. Penezić Z, Zarković M, Vujović S, et al. Value of assessing adrenocorticotropic hormone (ACTH) levels in differential diagnosis of hypercorticism. Med Pregl. 2004;57(7-8):335-342. doi: https://doi.org/10.2298/mpns0408335p

28. Olsen H, Kjellbom A, Löndahl M, Lindgren O. Suppressed ACTH Is Frequently Unrelated to Autonomous Cortisol Secretion in Patients With Adrenal Incidentalomas. J Clin Endocrinol Metab. 2019;104(2):506-512. doi: https://doi.org/10.1210/jc.2018-01029

29. Di Dalmazi $G$, Berr CM, Fassnacht $M$, et al. Adrenal function after adrenalectomy for subclinical hypercortisolism and Cushing's syndrome: a systematic review of the literature. J Clin Endocrinol Metab. 2014;99(8):2637-2645. doi: https://doi.org/10.1210/jc.2014-1401 
30. Prete A, Paragliola RM, Bottiglieri F, et al. Factors predicting the duration of adrenal insufficiency in patients successfully treated for Cushing disease and nonmalignant primary adrenal Cushing syndrome. Endocrine. 2017;55(3):969-980. doi: https://doi.org/10.1007/s12020-016-1007-5

31. Hurtado MD, Cortes T, Natt N, et al. Extensive clinical experience: Hypothalamic-pituitary-adrenal axis recovery after adrenalectomy for corticotropin-independent cortisol excess. Clin Endocrinol (Oxf). 2018;89(6):721-733. doi: https://doi.org/10.1111/cen.13803

32. Schalin-Jäntti C, Raade M, Hämäläinen E, Sane T. A 5-Year Prospective Follow-Up Study of Lipid-Rich Adrenal Incidentalomas: No Tumor Growth or Development of Hormonal Hypersecretion. Endocrinol Metab (Seoul). 2015;30(4):481-487. doi: https://doi.org/10.3803/EnM.2015.30.4.481

33. Lenders JW, Duh QY, Eisenhofer G, et al. Pheochromocytoma and paraganglioma: an endocrine society clinical practice guideline. J Clin Endocrinol Metab. 2014;99(6):1915-1942. doi: https://doi.org/10.1210/jc.2014-1498

34. Därr R, Kuhn M, Bode C, et al. Accuracy of recommended sampling and assay methods for the determination of plasma-free and urinary fractionated metanephrines in the diagnosis of pheochromocytoma and paraganglioma: a systematic review. Endocrine. 2017:56(3):495-503. doi: https://doi.org/10.1007/s12020-017-1300-y

35. Mullins F, O'Shea P, FitzGerald R, Tormey W. Enzyme-linked immunoassay for plasma-free metanephrines in the biochemical diagnosis of phaeochromocytoma in adults is not ideal. Clin Chem Lab Med. 2011;50(1):105-110. Published 2011 Oct 8. doi: https://doi.org/10.1515/CCLM.2011.742

36. Мельниченко Г.А., Трошина Е.А., Бельцевич Д.Г., и др. Клинические рекомендации Российской ассоциации эндокринологов по диагностике и лечению феохромоцитомы/параганглиомы // Эндокринная хирургия. - 2015. - Т. 9. — №3. - С. 15-33. [Melnichenko GA, Troshina EA, Beltsevich DG, et al. Russian Association of Endocrinologists clinical practice guidelines for diagnosis and treatment of pheochromocytoma and paraganglioma. Endocrine Surgery. 2015;9(3):15-33. (In Russ.)]. doi: https://doi.org/10.14341/serg2015315-33

37. Lenders JW, Pacak K, Walther MM, et al. Biochemical diagnosis of pheochromocytoma: which test is best? JAMA. 2002;287(11):1427-1434 doi: https://doi.org/10.1001/jama.287.11.1427

38. Sbardella E, Grossman AB. Pheochromocytoma: An approach to diagnosis. Best Pract Res Clin Endocrinol Metab. 2020;34(2):101346. doi: https://doi.org/10.1016/j.beem.2019.101346

39. Yu R, Wei M. False positive test results for pheochromocytoma from 2000 to 2008. Exp Clin Endocrinol Diabetes. 2010;118(9):577-585. doi: https://doi.org/10.1055/s-0029-1237699

40. Neary NM, King KS, Pacak K. Drugs and pheochromocytoma--don't be fooled by every elevated metanephrine. N Engl J Med. 2011;364(23):2268-2270. doi: https://doi.org/10.1056/NEJMc1101502\#SA1

41. Eisenhofer $\mathrm{G}$, Goldstein DS, Walther MM, et al. Biochemical diagnosis of pheochromocytoma: how to distinguish true- from falsepositive test results. J Clin Endocrinol Metab. 2003;88(6):2656-2666. doi: https://doi.org/10.1210/jc.2002-030005

42. Meyer-Rochow GY, Schembri GP, Benn DE, et al. The utility of metaiodobenzylguanidine single photon emission computed tomography/computed tomography (MIBG SPECT/CT) for the diagnosis of pheochromocytoma. Ann Surg Oncol. 2010;17(2):392-400. doi: https://doi.org/10.1245/s10434-009-0850-5

43. Derlin T, Busch JD, Wisotzki C, et al. Intraindividual comparison of 123I-mIBG SPECT/MRI, 123I-mIBG SPECT/CT, and MRI for the detection of adrenal pheochromocytoma in patients with elevated urine or plasma catecholamines. Clin Nucl Med. 2013;38(1):e1-e6. doi: https://doi.org/10.1097/RLU.0b013e318263923d

44. Sane T, Schalin-Jäntti C, Raade M. Is biochemical screening for pheochromocytoma in adrenal incidentalomas expressing low unenhanced attenuation on computed tomography necessary?. J Clin Endocrinol Metab. 2012;97(6):2077-2083. doi: https://doi.org/10.1210/jc.2012-1061

45. Buitenwerf E, Korteweg T, Visser A, et al. Unenhanced CT imaging is highly sensitive to exclude pheochromocytoma: a multicenter study. Eur J Endocrinol. 2018;178(5):431-437. doi: https://doi.org/10.1530/EJE-18-0006

46. Stowasser M. Update in primary aldosteronism. J Clin Endocrinol Metab. 2015;100(1):1-10. doi: https://doi.org/10.1210/jc.2014-3663
47. Milliez P, Girerd X, Plouin PF, et al. Evidence for an increased rate of cardiovascular events in patients with primary aldosteronism. J Am Coll Cardiol. 2005:45(8):1243-1248. doi: https://doi.org/10.1016/j.jacc.2005.01.015

48. Young WF Jr. Diagnosis and treatment of primary aldosteronism: practical clinical perspectives. J Intern Med. 2019;285(2):126-148. doi: https://doi.org/10.1111/joim.12831

49. Hiramatsu K, Yamada T, Yukimura Y, et al. A screening test to identify aldosterone-producing adenoma by measuring plasma renin activity. Results in hypertensive patients. Arch Intern Med. 1981;141(12):1589-1593.

50. McKenna TJ, Sequeira SJ, Heffernan A, et al. Diagnosis under random conditions of all disorders of the renin-angiotensin-aldosterone axis, including primary hyperaldosteronism. J Clin Endocrinol Metab. 1991;73(5):952-957. doi: https://doi.org/10.1210/jcem-73-5-952

51. Stowasser M, Gordon RD. Primary aldosteronism-careful investigation is essential and rewarding. Mol Cell Endocrinol. 2004;217(1-2):33-39. doi: https://doi.org/10.1016/j.mce.2003.10.006

52. Veldhuizen GP, Alnazer RM, Kroon AA, de Leeuw PW. Confounders of the aldosterone-to-renin ratio when used as a screening test in hypertensive patients: A critical analysis of the literature. J Clin Hypertens (Greenwich). 2021;23(2):201-207. doi: https://doi.org/10.1111/jch.14117

53. O'Shea PM, Griffin TP, Denieffe S, Fitzgibbon MC. The aldosterone to renin ratio in the diagnosis of primary aldosteronism: Promises and challenges. Int J Clin Pract. 2019;73(7):e13353. doi: https://doi.org/10.1111/ijcp.13353

54. Schilbach K, Junnila RK, Bidlingmaier M. Aldosterone to Renin Ratio as Screening Tool in Primary Aldosteronism. Exp Clin Endocrinol Diabetes. 2019;127(2-03):84-92. doi: https://doi.org/10.1055/a-0672-0836

55. Stowasser M, Ahmed AH, Pimenta E, et al. Factors affecting the aldosterone/renin ratio. Horm Metab Res. 2012;44(3):170-176. doi: https://doi.org/10.1055/s-0031-1295460

56. Widimský J, Bruthans J, Wohlfahrt P, et al. Primary aldosteronism in a general population sample. The Czech post-MONICA study. Blood Press. 2020;29(3):191-198. doi: https://doi.org/10.1080/08037051.2020.1723406

57. Douillard C, Houillier P, Nussberger J, Girerd X. SFE/SFHTA AFCE Consensus on Primary Aldosteronism, part 2: First diagnostic steps. Ann Endocrinol (Paris). 2016;77(3):192-201. doi: https://doi.org/10.1016/j.ando.2016.02.003

58. Young WF. Primary aldosteronism: renaissance of a syndrome. Clin Endocrinol (Oxf). 2007;66(5):607-618. doi: https://doi.org/10.1111/j.1365-2265.2007.02775.x

59. Mosso L, Carvajal C, González A, et al. Primary aldosteronism and hypertensive disease. Hypertension. 2003:42(2):161-165. doi: https://doi.org/10.1161/01.HYP.0000079505.25750.11

60. Funder JW, Carey RM, Mantero F, et al. The Management of Primary Aldosteronism: Case Detection, Diagnosis, and Treatment: An Endocrine Society Clinical Practice Guideline. J Clin Endocrinol Metab. 2016;101(5):1889-1916. doi: https://doi.org/10.1210/jc.2015-4061

61. Mulatero P, Stowasser M, Loh KC, et al. Increased diagnosis of primary aldosteronism, including surgically correctable forms, in centers from five continents. J Clin Endocrinol Metab. 2004;89(3):1045-1050. doi: https://doi.org/10.1210/jc.2003-031337

62. Monticone S, Burrello J, Tizzani D, et al. Prevalence and Clinical Manifestations of Primary Aldosteronism Encountered in Primary Care Practice. J Am Coll Cardiol. 2017;69(14):1811-1820. doi: https://doi.org/10.1016/j.jacc.2017.01.052

63. Kokko E, Nevalainen $\mathrm{Pl}$, Choudhary MK, et al. Aldosteroneto-renin ratio is related to arterial stiffness when the screening criteria of primary aldosteronism are not met. Sci Rep. 2020;10(1):19804. Published 2020 Nov 13 doi: https://doi.org/10.1038/s41598-020-76718-7

64. Kisaka T, Ozono R, Ishida T, et al. Association of elevated plasma aldosterone-to-renin ratio with future cardiovascular events in patients with essential hypertension. $J$ Hypertens 2012:30(12):2322-2330. doi:10.1097/HJH.0b013e328359862d

65. Shapiro Y, Boaz M, Matas Z, et al. The association between the renin-angiotensin-aldosterone system and arterial stiffness in young healthy subjects. Clin Endocrinol (Oxf). 2008;68(4):510-512. doi: https://doi.org/10.1111/j.1365-2265.2008.03176.x

66. Cain JP, Tuck ML, Williams GH, et al. The regulation of aldosterone secretion in primary aldosteronism. Am J Med. 1972;53(5):627-637. doi: https://doi.org/10.1016/0002-9343(72)90158-1 
67. d'Alva CB, Abiven-Lepage G, Viallon V, et al. Sex steroids in androgen-secreting adrenocortical tumors: clinical and hormonal features in comparison with non-tumoral causes of androgen excess. Eur J Endocrinol. 2008;159(5):641-647. doi: https://doi.org/10.1530/EJE-08-0324

68. Di Dalmazi G. Hyperandrogenism and Adrenocortical Tumors. Front Horm Res. 2019;53:92-99. doi: https://doi.org/10.1159/000494905

69. Fassnacht M, Kroiss M, Allolio B. Update in adrenocortical carcinoma. J Clin Endocrinol Metab. 2013;98(12):4551-4564. doi: https://doi.org/10.1210/jc.2013-3020

70. Chortis V, Bancos I, Nijman T, et al. Urine Steroid Metabolomics as a Novel Tool for Detection of Recurrent Adrenocortical Carcinoma. J Clin Endocrinol Metab. 2020;105(3):e307-e318. doi: https://doi.org/10.1210/clinem/dgz141

71. Claahsen-van der Grinten HL, Hermus AR, Otten BJ. Testicular adrenal rest tumours in congenital adrenal hyperplasia. Int J Pediatr Endocrinol. 2009;2009:624823. doi: https://doi.org/10.1155/2009/624823

72. Bourdeau I, El Ghorayeb N, Gagnon N, Lacroix A. MANAGEMENT OF ENDOCRINE DISEASE: Differential diagnosis, investigation and therapy of bilateral adrenal incidentalomas. Eur J Endocrinol. 2018;179(2):R57-R67. doi: https://doi.org/10.1530/EJE-18-0296

73. Шевэ А., Селиванова Л.С., Кузнецов Н.С., и др. Иммуногистохимическое исследование экспрессии аберрантных/эутопических рецепторов у пациентов с макронодулярной гиперплазией надпочечников // Проблемы эндокринологии. - 2020. - Т. 66. - №6. - C. 4-12. [Chevais A, Selivanova LS, Kuznetsov NS, et al. Immunohistochemical study on the expression/hyperexpression of aberrant/eutopic receptors in patients with bilateral macronodular adrenal hyperplasia. Problems of Endocrinology. 2020;66(6):4-12. (In Russ.)]. doi: https://doi.org/10.14341/probl12516

74. Bornstein SR, Allolio B, Arlt W, et al. Diagnosis and Treatment of Primary Adrenal Insufficiency: An Endocrine Society Clinical Practice Guideline. J Clin Endocrinol Metab. 2016;101(2):364-389. doi: https://doi.org/10.1210/jc.2015-1710

75. Del Monte P, Bernasconi D, Bertolazzi L, et al. Increased 17 alpha-hydroxyprogesterone response to ACTH in silent adrenal adenoma: cause or effect?. Clin Endocrinol (Oxf). 1995;42(3):273-277. doi: https://doi.org/10.1111/j.1365-2265.1995.tb01875.x

76. Jaresch S, Kornely E, Kley HK, Schlaghecke R. Adrenal incidentaloma and patients with homozygous or heterozygous congenital adrenal hyperplasia. J Clin Endocrinol Metab. 1992;74(3):685-689. doi: https://doi.org/10.1210/jcem.74.3.1311000

77. Speiser PW, Arlt W, Auchus RJ, et al. Congenital Adrenal Hyperplasia Due to Steroid 21-Hydroxylase Deficiency: An Endocrine Society Clinical Practice Guideline [published correction appears in J Clin Endocrinol Metab. 2019 Jan 1;104(1):39-40]. J Clin Endocrinol Metab. 2018;103(11):4043-4088. doi: https://doi.org/10.1210/jc.2018-01865

78. Al-Hawary MM, Francis IR, Korobkin M. Non-invasive evaluation of the incidentally detected indeterminate adrenal mass. Best Pract Res Clin Endocrinol Metab. 2005;19(2):277-292. doi: https://doi.org/10.1016/j.beem.2004.09.002

79. McCarthy CJ, McDermott S, Blake MA. Adrenal Imaging: Magnetic Resonance Imaging and Computed Tomography. Front Horm Res. 2016:45:55-69. doi: https://doi.org/10.1159/000442313

80. Warda MH, Shehata SM, Zaiton F. Chemical-shift MRI versus washout CT for characterizing adrenal incidentalomas. Clin Imaging. 2016;40(4):780-787. doi: https://doi.org/10.1016/j.clinimag.2016.02.006

81. Hamrahian AH, loachimescu AG, Remer EM, et al. Clinical utility of noncontrast computed tomography attenuation value (hounsfield units) to differentiate adrenal adenomas/hyperplasias from nonadenomas: Cleveland Clinic experience. J Clin Endocrinol Metab. 2005;90(2):871-877. doi: https://doi.org/10.1210/jc.2004-1627

82. Dinnes J, Bancos I, Ferrante di Ruffano L, et al. MANAGEMENT OF ENDOCRINE DISEASE: Imaging for the diagnosis of malignancy in incidentally discovered adrenal masses: a systematic review and meta-analysis. Eur J Endocrinol. 2016;175(2):R51-R64. doi: https://doi.org/10.1530/EJE-16-0461

83. Sabet FA, Majdzadeh R, Mostafazadeh Davani B, Heidari K, Soltani A. Likelihood ratio of computed tomography characteristics for diagnosis of malignancy in adrenal incidentaloma: systematic review and meta-analysis. J Diabetes Metab Disord. 2016;15:12. Published 2016 Apr 21. doi: https://doi.org/10.1186/s40200-016-0224-z
84. Terzolo M, Reimondo G, Bovio S, Angeli A. Subclinical Cushing's syndrome. Pituitary. 2004;7(4):217-223. doi: https://doi.org/10.1007/s11102-005-4024-6

85. Fan J, Tang J, Fang J, et al. Ultrasound imaging in the diagnosis of benign and suspicious adrenal lesions. Med Sci Monit. 2014;20:2132-2141. Published 2014 Nov 3. doi: https://doi.org/10.12659/MSM.890800

86. Petersenn S, Richter PA, Broemel T, et al. Computed tomography criteria for discrimination of adrenal adenomas and adrenocortical carcinomas: analysis of the German ACC registry. Eur J Endocrinol. 2015;172(4):415-422. doi: https://doi.org/10.1530/EJE-14-0916

87. Cistaro A, Niccoli Asabella A, Coppolino P, et al. Diagnostic and prognostic value of 18F-FDG PET/CT in comparison with morphological imaging in primary adrenal gland malignancies a multicenter experience. Hell J Nucl Med. 2015;18(2):97-102. doi: https://doi.org/10.1967/s002449910202

88. Guerin C, Pattou F, Brunaud L, et al. Performance of 18F-FDG PET/CT in the Characterization of Adrenal Masses in Noncancer Patients: A Prospective Study. J Clin Endocrinol Metab. 2017;102(7):2465-2472. doi: https://doi.org/10.1210/jc.2017-00254

89. Nakajo M, Jinguji M, Nakajo $M$, et al. Texture analysis of FDG PET/CT for differentiating between FDG-avid benign and metastatic adrenal tumors: efficacy of combining SUV and texture parameters. Abdom Radiol (NY). 2017;42(12):2882-2889. doi: https://doi.org/10.1007/s00261-017-1207-3

90. Kim SJ, Lee SW, Pak K, et al. Diagnostic accuracy of 18F-FDG PET or PET/CT for the characterization of adrenal masses: a systematic review and meta-analysis. Br J Radiol. 2018;91(1086):20170520. doi: https://doi.org/10.1259/bjr.20170520

91. Timmers HJ, Chen CC, Carrasquillo JA, et al. Comparison of 18F-fluoro-L-DOPA, 18F-fluoro-deoxyglucose, and 18F-fluorodopamine PET and 123I-MIBG scintigraphy in the localization of pheochromocytoma and paraganglioma. J Clin Endocrinol Metab. 2009;94(12):4757-4767. doi: https://doi.org/10.1210/jc.2009-1248

92. Alencar GA, Fragoso MC, Yamaga LY, et al. (18)F-FDG-PET/CT imaging of ACTH-independent macronodular adrenocortical hyperplasia (AIMAH) demonstrating increased (18)F-FDG uptake. J Clin Endocrinol Metab. 2011;96(11):3300-3301. doi: https://doi.org/10.1210/jc.2011-1397

93. Arellano RS, Harisinghani MG, Gervais DA, et al. Image-guided percutaneous biopsy of the adrenal gland: review of indications, technique, and complications. Curr Probl Diagn Radiol. 2003;32(1):3-10. doi: https://doi.org/10.1067/cdr.2003.120002

94. Harisinghani MG, Maher MM, Hahn PF, et al. Predictive value of benign percutaneous adrenal biopsies in oncology patients. Clin Radiol. 2002;57(10):898-901. doi: https://doi.org/10.1053/crad.2002.1054

95. Bancos I, Tamhane S, Shah M, et al. DIAGNOSIS OF ENDOCRINE DISEASE: The diagnostic performance of adrenal biopsy: a systematic review and meta-analysis. Eur J Endocrinol. 2016;175(2):R65-R80. doi: https://doi.org/10.1530/EJE-16-0297

96. Молашенко Н.В., Платонова Н.М., Бельцевич Д.Г., и др. Дифференциальная диагностика инциденталом надпочечников // Ожирение и метаболизм. - 2016. T. 13. - №.4 - C. 39-44. [Molashenko NV, Platonova NM, Beltsevich DG, et al. Diagnosis and differential diagnosis of adrenal incidentalomas. Obesity and metabolism. 2016;13(4):39-44. (In Russ.)] doi: 10.14341/OMET2016439-44

97. Anagnostis P, Efstathiadou Z, Polyzos SA, et al. Long term follow-up of patients with adrenal incidentalomas a single center experience and review of the literature. Exp Clin Endocrinol Diabetes. 2010;118(9):610-616. doi: https://doi.org/10.1055/s-0029-1237704

98. Yilmaz H, Tütüncü NB, Sahin M. Two-year follow-up of thirty-two non-functional benign adrenal incidentalomas. J Endocrinol Invest 2009;32(11):913-916. doi: https://doi.org/10.1007/BF03345772

99. Brix D, Allolio B, Fenske W, et al. Laparoscopic versus open adrenalectomy for adrenocortical carcinoma: surgical and oncologic outcome in 152 patients. Eur Urol. 2010;58(4):609-615. doi: https://doi.org/10.1016/j.eururo.2010.06.024

100. Cooper AB, Habra MA, Grubbs EG, et al. Does laparoscopic adrenalectomy jeopardize oncologic outcomes for patients with adrenocortical carcinoma?. Surg Endosc. 2013;27(11):4026-4032. doi: https://doi.org/10.1007/s00464-013-3034-0 
101. Donatini G, Caiazzo R, Do Cao C, et al. Long-term survival after adrenalectomy for stage $\mathrm{I} / \mathrm{I}$ adrenocortical carcinoma (ACC): a retrospective comparative cohort study of laparoscopic versus open approach. Ann Surg Oncol. 2014;21(1):284-291 doi: https://doi.org/10.1245/s10434-013-3164-6

102. Fosså $A$, Røsok Bl, Kazaryan AM, et al. Laparoscopic versus open surgery in stage $\mathrm{I}-\mathrm{III}$ adrenocortical carcinoma -- a retrospective comparison of 32 patients. Acta Oncol. 2013;52(8):1771-1777 doi: https://doi.org/10.3109/0284186X.2013.765065

103. Lombardi CP, Raffaelli M, De Crea C, et al. Open versus endoscopic adrenalectomy in the treatment of localized (stage I/II) adrenocortical carcinoma: results of a multiinstitutional Italian survey. Surgery. 2012;152(6):1158-1164. doi: https://doi.org/10.1016/j.surg.2012.08.014

104. Miller BS, Ammori JB, Gauger PG, Broome JT, Hammer GD, Doherty GM. Laparoscopic resection is inappropriate in patients with known or suspected adrenocortical carcinoma. World J Surg. 2010:34(6):1380-1385 doi: https://doi.org/10.1007/s00268-010-0532-2

105. Miller BS, Gauger PG, Hammer GD, Doherty GM. Resection of adrenocortical carcinoma is less complete and local recurrence occurs sooner and more often after laparoscopic adrenalectomy than after open adrenalectomy. Surgery. 2012;152(6):1150-1157. doi: https://doi.org/10.1016/j.surg.2012.08.024

106. Mir MC, Klink JC, Guillotreau J, et al. Comparative outcomes of laparoscopic and open adrenalectomy for adrenocortical carcinoma: single, high-volume center experience. Ann Surg Oncol. 2013;20(5):1456-1461. doi: https://doi.org/10.1245/s10434-012-2760-

107. Porpiglia F, Fiori C, Daffara F, et al. Retrospective evaluation of the outcome of open versus laparoscopic adrenalectomy for stage I and II adrenocortical cancer. Eur Urol. 2010;57(5):873-878. doi: https://doi.org/10.1016/j.eururo.2010.01.036

108. Langenhuijsen J, Birtle A, Klatte T, et al. Surgical Management of Adrenocortical Carcinoma: Impact of Laparoscopic Approach, Lymphadenectomy, and Surgical Volume on Outcomes-A Systematic Review and Meta-analysis of the Current Literature [published correction appears in Eur Urol Focus. 2018 Apr;4(3):461]. Eur Urol Focus. 2016;1(3):241-250. doi: https://doi.org/10.1016/j.euf.2015.12.001

109. Morelli V, Minelli L, Eller-Vainicher C, et al. Predictability of hypoadrenalism occurrence and duration after adrenalectomy for ACTH-independent hypercortisolism. J Endocrinol Invest. 2018;41(4):485-493. doi: https://doi.org/10.1007/s40618-017-0788-6
110. Debillon E, Velayoudom-Cephise FL, Salenave S, et al. Unilateral Adrenalectomy as a First-Line Treatment of Cushing's Syndrome in Patients With Primary Bilateral Macronodular Adrenal Hyperplasia. J Clin Endocrinol Metab. 2015;100(12):4417-4424. doi: https://doi.org/10.1210/jc.2015-2662

111. Osswald A, Quinkler M, Di Dalmazi G, et al. Long-Term Outcome of Primary Bilateral Macronodular Adrenocortical Hyperplasia After Unilateral Adrenalectomy. J Clin Endocrinol Metab. 2019;104(7):2985-2993. doi: https://doi.org/10.1210/jc.2018-02204

112. Sheikh-Ahmad M, Dickstein G, Matter I, et al. Unilateral Adrenalectomy for Primary Bilateral Macronodular Adrenal Hyperplasia: Analysis of 71 Cases. Exp Clin Endocrinol Diabetes. 2020;128(12):827-834. doi: https://doi.org/10.1055/a-0998-7884

113. Xu Y, Rui W, Qi Y, et al. The role of unilateral adrenalectomy in corticotropin-independent bilateral adrenocortical hyperplasias. World J Surg. 2013;37(7):1626-1632 doi: https://doi.org/10.1007/s00268-013-2059-9

114. Bernini GP, Moretti A, Oriandini C, et al. Long-term morphological and hormonal follow-up in a single unit on 115 patients with adrenal incidentalomas. Br J Cancer. 2005;92(6):1104-1109. doi: https://doi.org/10.1038/sj.bjc.6602459

115. Barzon L, Scaroni C, Sonino N, et al. Risk factors and long-term follow-up of adrenal incidentalomas. J Clin Endocrinol Metab. 1999;84(2):520-526. doi: https://doi.org/10.1210/jcem.84.2.5444

116. Giordano R, Marinazzo E, Berardelli R, et al. Long-term morphological, hormonal, and clinical follow-up in a single unit on 118 patients with adrenal incidentalomas. Eur J Endocrinol. 2010;162(4):779-785. doi: https://doi.org/10.1530/EJE-09-0957

117. Barzon L, Sonino N, Fallo F, et al. Prevalence and natural history of adrenal incidentalomas. Eur J Endocrinol. 2003;149(4):273-285. doi: https://doi.org/10.1530/eje.0.1490273

118. Berland LL, Silverman SG, Gore RM, et al. Managing incidental findings on abdominal CT: white paper of the ACR incidental findings committee. J Am Coll Radiol. 2010;7(10):754-773. doi: https://doi.org/10.1016/j.jacr.2010.06.013

119. Eisenhauer EA, Therasse P, Bogaerts J, et al. New response evaluation criteria in solid tumours: revised RECIST guideline (version 1.1). Eur J Cancer. 2009;45(2):228-247. doi: https://doi.org/10.1016/j.ejca.2008.10.026

120. Elamin MB, Murad MH, Mullan R, et al. Accuracy of diagnostic tests for Cushing's syndrome: a systematic review and metaanalyses. J Clin Endocrinol Metab. 2008;93(5):1553-1562. doi: https://doi.org/10.1210/jc.2008-0139

ПРИЛОЖЕНИЕ А

МЕТОДОЛОГИЯ РАЗРАБОТКИ КЛИНИЧЕСКИХ РЕКОМЕНДАЦИЙ

Таблица 1. Шкала оценки уровней достоверности доказательств (УДД) для методов диагностики (диагностических вмешательств)

уДД Расшифровка

1 Систематические обзоры исследований с контролем референсным методом или систематический обзор рандомизированных клинических исследований с применением метаанализа

Отдельные исследования с контролем референсным методом или отдельные рандомизированные

2 клинические исследования и систематические обзоры исследований любого дизайна, за исключением рандомизированных клинических исследований, с применением метаанализа

Исследования без последовательного контроля референсным методом или исследования

3 с референсным методом, не являющимся независимым от исследуемого метода, или нерандомизированные сравнительные исследования, в том числе когортные исследования

4 Несравнительные исследования, описание клинического случая 
Таблица 2. Шкала оценки уровней достоверности доказательств (УДД) для методов профилактики, лечения и реабилитации (профилактических, лечебных, реабилитационных вмешательств)

\begin{tabular}{cl}
\hline УдД & \multicolumn{1}{c}{ Расшифровка } \\
\hline 1 & Систематический обзор РКИ с применением метаанализа \\
\hline 2 & $\begin{array}{l}\text { Отдельные РКИ и систематические обзоры исследований любого дизайна, за исключением РКИ, с } \\
\text { применением метаанализа }\end{array}$ \\
\hline 3 & Нерандомизированные сравнительные исследования, в т.ч. когортные исследования \\
\hline 4 & $\begin{array}{l}\text { Несравнительные исследования, описание клинического случая или серии случаев, исследования } \\
\text { «случай-контроль» }\end{array}$ \\
\hline 5 & $\begin{array}{l}\text { Имеется лишь обоснование механизма действия вмешательства (доклинические исследования) или } \\
\text { мнение экспертов }\end{array}$ \\
\hline
\end{tabular}

Таблица 3. Шкала оценки уровней убедительности рекомендаций (УУР) для методов профилактики, диагностики, лечения и реабилитации (профилактических, диагностических, лечебных, реабилитационных вмешательств)

\begin{tabular}{cl}
\hline уУР & \multicolumn{1}{c}{ Расшифровка } \\
\hline A & $\begin{array}{l}\text { Сильная рекомендация (все рассматриваемые критерии эффективности (исходы) являются важными, } \\
\text { все исследования имеют высокое или удовлетворительное методологическое качество, их выводы по } \\
\text { интересующим исходам являются согласованными) }\end{array}$ \\
\hline В & $\begin{array}{l}\text { Условная рекомендация (не все рассматриваемые критерии эффективности (исходы) являются важными, } \\
\text { не все исследования имеют высокое или удовлетворительное методологическое качество и/или их } \\
\text { выводы по интересующим исходам не являются согласованными) }\end{array}$ \\
\hline C & $\begin{array}{l}\text { Слабая рекомендация (отсутстие доказательств надлежащего качества (все рассматриваемые критерии } \\
\text { эффективнсти (исходы) являются неважными, все исследования имеют низкое методологическое } \\
\text { качество и их выводы по интересующим исходам не являются согласованными) }\end{array}$ \\
\hline
\end{tabular}

ПРИЛОЖЕНИЕ Б.

АЛГОРИТМЫ ДЕЙСТВИЙ ВРАЧА

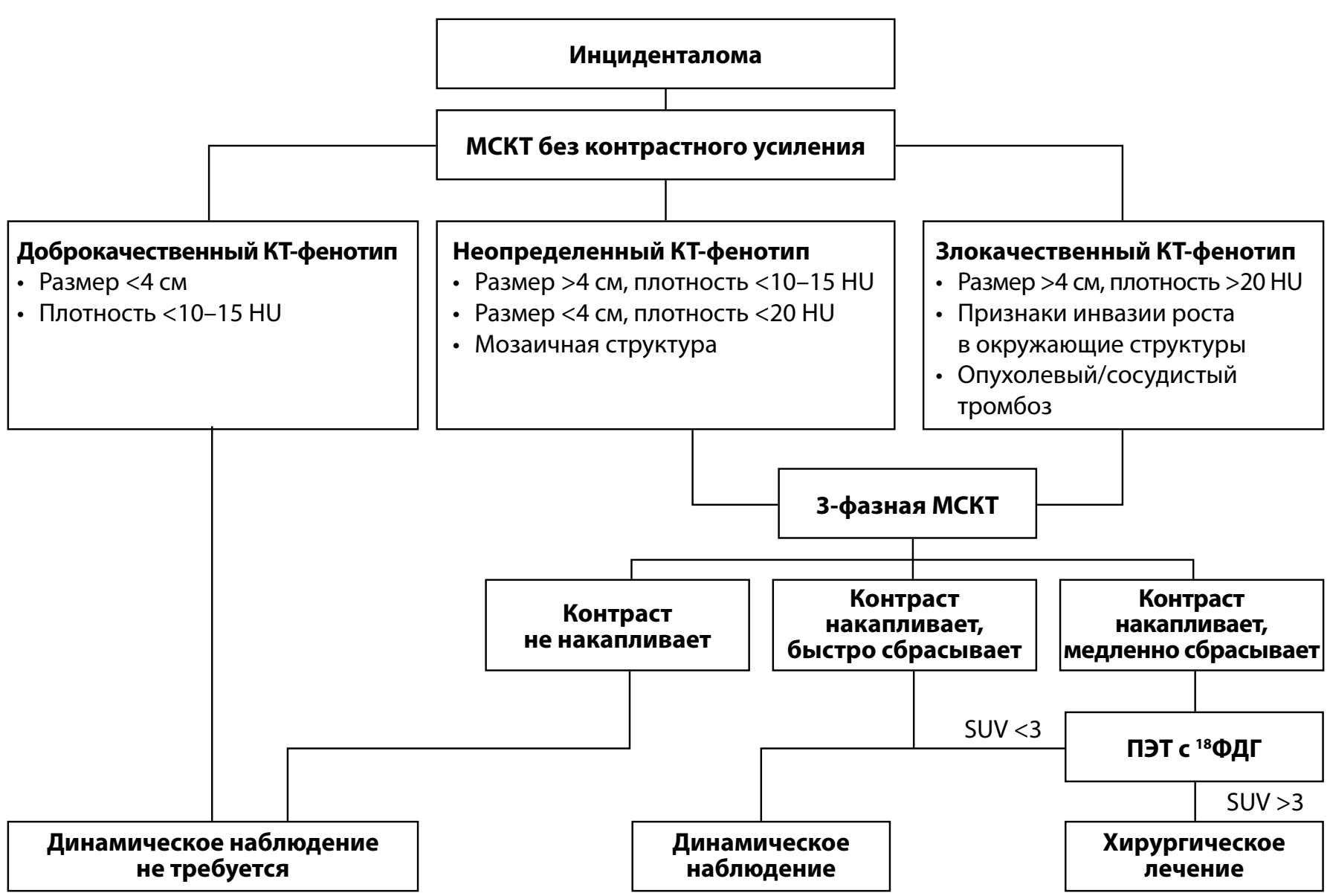




\section{ИНФОРМАЦИЯ ОБ АВТОРАХ [AUTHORS INFO]}

*Шевэ Анастасия, аспирант [Anastassia Chevais, postgraduate student], адрес: Россия, 117036, Москва, ул. Дм. Ульянова, д. 11 [address: 11 Dmitry Ulyanova street, 117036 Moscow, Russia]. Тел.: +7-962-993-27-63; ORCID: https://orcid.org/0000-0001-5592-4794; eLibrary SPIN: 2459-0540; e-mail: anastassia93@gmail.com

Бельцевич Дмитрий Германович, д.м.н., профессор [Dmitriy G. Beltsevich, MD, PhD, Professor]; ORCID: https://orcid.org/0000-0001-7098-4584; eLibrary SPIN: 4475-6327; e-mail: belts67@gmail.com Трошина Екатерина Анатольевна, д.м.н., профессор, член-корреспондент PAH [Ekaterina A. Troshina, MD, PhD, professor]; ORCID: https://orcid.org/0000-0002-8520-8702; eLibrary SPIN: 8821-8990; e-mail: troshina@inbox.ru Мельниченко Галина Афанасьевна, д.М.н., профессор, академик PAH [Galina A. Melnichenko, MD, PhD, Professor]; ORCID: http://orcid.org/0000-0002-5634-7877; eLibrary SPIN: 8615-0038; e-mail: teofrast2000@mail.ru

Платонова Надежда Михайловна, д.м.н. [Nadezhda M. Platonova, MD, ScD];

ORCID: https://orcid.org/0000-0001-6388-1544; eLibrary SPIN: 4053-3033; e-mail: doc-platonova@inbox.ru

Ладыгина Дарья Олеговна, K.M.H. [Daria O. Ladygina, MD, PhD]; ORCID: http://orcid.org/0000-0001-6418-7060; eLibrary SPIN: 7958-9435; e-mail: ladygina.do@gmail.com

\section{ИНФОРМАЦИЯ}

Рукопись получена: 01.09.2021. Одобрена к публикации: 20.09.2021

\section{ЦИТИРОВАТЬ:}

Бельцевич Д.Г., Трошина Е.А., Мельниченко Г.А., Платонова Н.М., Ладыгина Д.О., Шевэ А. Проект клинических рекомендаций «Инциденталома надпочечника» // Эндокринная хирургия. — 2021. — Т. 15. — №1. — С. 4-26 doi: https://doi.org/10.14341/serg12712

\section{TO CITE THIS ARTICLE:}

Beltsevich DG, Troshina EA, Melnichenko GA, Platonova NM, Ladygina DO, Chevais A. Draft of the clinical practice guidelines "Adrenal incidentaloma". Endocrine surgery. 2021;15(1):4-26. doi: https://doi.org/10.14341/serg12712 